\title{
Spatial crowdsourcing with mobile agents in vehicular networks
}

\author{
Oscar Urra ${ }^{a, *}$, Sergio Ilarri ${ }^{\mathrm{a}, \mathrm{b}}$ \\ a Department of Computer Science and Systems Engineering, University of Zaragoza, Zaragoza, Spain \\ b I3A, University of Zaragoza, Zaragoza, Spain
}

\section{A R T I C L E I N F O}

\section{Article history:}

Received 5 October 2018

Received in revised form 10 February 2019

Accepted 20 March 2019

Available online $\mathrm{xxxx}$

\section{Keywords:}

Vehicular ad hoc networks

Spatial crowdsourcing

Mobile agents

Data management

Environment monitoring

\begin{abstract}
A B S T R A C T
In the last years, the automotive industry has shown interest in the addition of computing and communication devices to cars, thanks to technological advances in these fields, in order to meet the increasing demand of "connected" applications and services. Although vehicular ad hoc networks (VANETs) have not been fully developed yet, they could be used in a near future as a means to provide a number of interesting applications and services that need the exchange of data among vehicles and other data sources.

In this paper, we propose a spatial crowdsourcing schema for the opportunistic collection of information within an interest area in a city or region (e.g., measures about the environment, such as the concentration of certain gases in the atmosphere, or information such as the availability of parking spaces in an area), using vehicular ad hoc communications. We present a method that exploits mobile agent technology to accomplish the distributed collection and querying of data among vehicles in such a scenario. Our proposal is supported by an extensive set of realistic simulations that prove the feasibility of the approach.
\end{abstract}

(C) 2019 Published by Elsevier Inc.

\section{Introduction}

The continuous development of information and telecommunication technologies has brought advances to an increasing number of fields in industry. The automotive sector is not an exception and today's cars are more secure, respectful with the environment, and energy-efficient than never before, thanks to the inclusion of a wide range of computer devices and sensors. In this context, it is very relevant to provide cars with communication abilities so that they can exchange information with other cars (vehicle-to-vehicle communications) as well as with the road-side infrastructure and with other actors outside the roads (vehicle-toinfrastructure communications and vehicle-to-pedestrian communications). To accomplish this, the concept of vehicular network (also called vehicular ad hoc network or VANET) [1-3] has been proposed, as a set of vehicles driving along the roads of a certain geographic area, that can send/receive data to/from their neighbors by using short-range wireless communication devices. Vehicular networks open up many opportunities for the development of interesting applications, but they also imply a number of significant challenges from a data management perspective [4].

\footnotetext{
* Corresponding author.

E-mail addresses: ourra@itainnova.es (O. Urra), silarri@unizar.es (S. Ilarri).
}

According to [5], luxury cars have more than 100 sensors. If conventional cars were equipped with appropriate sensors (e.g., temperature, humidity, pollution gases, etc.) to obtain information from their surroundings, and given that they are constantly moving along wide areas and that the number of vehicles could be rather high, the amount of information that can be retrieved and stored in this way could be considerable. Additionally, if the owners of those vehicles are willing to share those data and a suitable mechanism exists to manage and process them properly, then it would be possible to generate knowledge that could be used as a basis for the creation of other value-added services. Moreover, some vehicles could physically move to a spatial area to measure certain environment parameters there or just to bring a specific query processing task to that area (i.e., to transport some code for its execution in that area). This form of spatial crowdsourcing could be used, for example, to obtain driving patterns from the vehicles belonging to the VANET, based on the hour of the day or the weather conditions, and thus offer to the drivers the most optimal route to reach their workplace. Alternatively, as another example, this ability could be exploited for surveillance or environment monitoring (e.g., to measure the pollution or noise level in different areas of a city). Moreover, according to a report by ABI Research [6,7], the use of crowdsourcing to obtain data by connected, sensor-equipped vehicles can be used to improve already-existing applications (e.g., parking or traffic-related services), and can also bring new business 


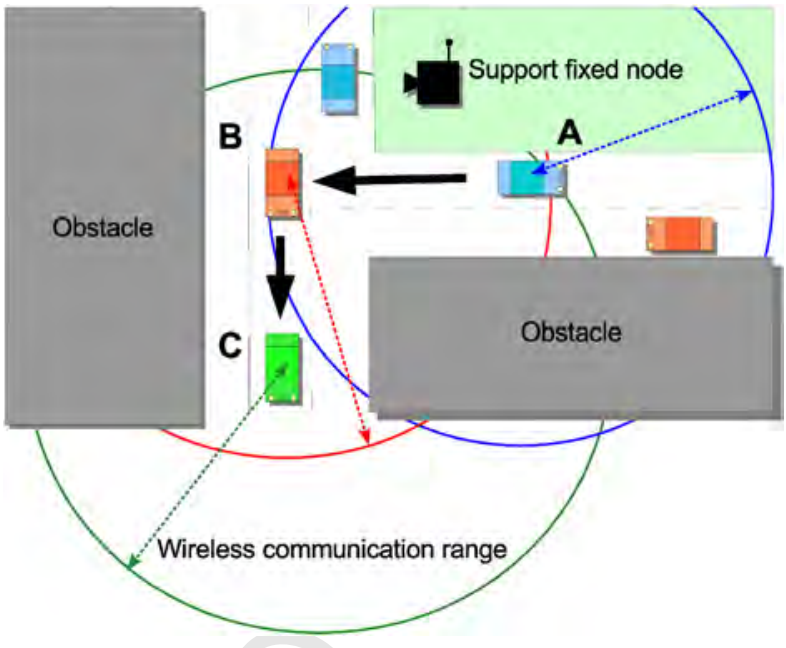

Fig. 1. Example of a VANET including a support fixed node and obstacles/buildings. (For interpretation of the colors in the figure(s), the reader is referred to the web version of this article.)

included in this paper shows the feasibility and the interest of the presented approach.

As an example of use case where the proposed approach can be applied, we can consider the case of air quality monitoring using low-cost air quality sensors aboard vehicles. For example, in the context of the European Project TRAFAIR - Understanding Traffic Flows to Improve Air quality (http://trafair.eu), one of the activities of the project tackles the deployment of an urban sensor network in several cities in Italy and Spain. In a scenario where vehicles carry air monitoring sensors, then it would be possible to perform flexible monitoring tasks to track air quality data about any area of the cities in a flexible way (rather than considering fixed monitoring areas covered by static sensors). In general, the spatial crowdsourcing approach described in this paper can be applied in any scenario where we need to monitor the environment by exploiting vehicles' sensors (e.g., for surveillance purposes [10], to create noise maps of the city [11], to obtain information about the availability of road-side parking spaces [12], etc.). As an example, SoundCity [13] is an Android application for mobile users, developed by Inria, that allows to measure one's personal exposure to noise pollution; however, it has not been developed for vehicular networks.

The rest of this paper is structured as follows. In Section 2, we describe basic background concerning intelligent vehicles, vehicular networks, and mobile agents. In Section 3, we describe a system for monitoring and querying data by using a VANET, as well as the use of spatial crowdsourcing techniques to improve the monitoring process. In Section 4, we perform several experiments to evaluate the performance of the proposed spatial crowdsourcing approach. In Section 5, we present some related work. Finally, in Section 6, we summarize our conclusions and present some lines of future work.

\section{Technological context}

In this section, we describe the background technologies that are used in our spatial crowdsourcing approach. Firstly, in Section 2.1, we focus on vehicular networks. Secondly, in Section 2.2 we describe the basics of mobile agent technology.

\subsection{Vehicular networks}

Enhancing existing vehicles with autonomous behavior and intelligent features, which has given rise to the concept of intelligent vehicles, is a relevant ingredient for the development of the socalled Intelligent Transportation Systems (ITS) [14,15]. These vehicles have the capability to sense the environment and communicate among themselves to share data relevant to the driver. A possibility to communicate these data is the use of cellular communications (e.g., 3G/4G), but they depend on a mobile telephony infrastructure, which does not cover all the areas (for example, inside tunnels and in certain shadow areas where the available coverage is limited) and have an economic cost that must be paid by the tions are publicly-available wireless networks deployed in certain cities by their local councils, but these are not present in all the cities and they usually do not cover all the city area either. Finally, we could consider the use of ad hoc communications, as an easy and non-expensive way of sharing resources would encourage the needed cooperation among users.

A Vehicular Ad Hoc Network (VANET) [2,3,16] is a highlymobile network whose nodes are vehicles traveling along roads or highways. In a VANET, vehicles can establish connections with other nearby vehicles and, in this way, they can exchange different types of information. This makes the development of new applications relevant to drivers (as well as passengers) possible, such as applications related to security, monitoring, entertainment, or data sharing. The link between those nodes is established directly in a peer-to-peer way by using any of the available short-range wireless communication technologies (such as Wi-Fi based on the $802.11 \mathrm{~b} / \mathrm{g} / \mathrm{n}$ standard or the more specific $802.11 \mathrm{p}$, WiMAX, Bluetooth, Zigbee, etc.); specifically, the current communications standard for VANETs is WAVE $[17,18]$.

Whereas VANETs open up new opportunities for the development of interesting applications, they also require the design of appropriate techniques from the perspective of data management [4]. Most difficulties are due to the high mobility of vehicles and the limited amount of time during which two vehicles may be within communication range of each other. Since the vehicles are constantly moving (usually at high speeds) and the wireless signal can be blocked by buildings and other obstacles, these connections may last only for a few seconds and therefore the network topology is constantly changing. For example, when a vehicle needs to communicate data to a specific target vehicle, a multihop routing protocol [19] must be usually used (e.g., see Fig. 1, where vehicle $B$ acts an intermediate relay point for the data sent from vehicle $A$ to vehicle $C$ ).

\subsection{Mobile agents}

A mobile agent [20-22] is a software program (composed by code and data) that can move from one computer or mobile deuser to the mobile phone operator company. Other alternative op- 


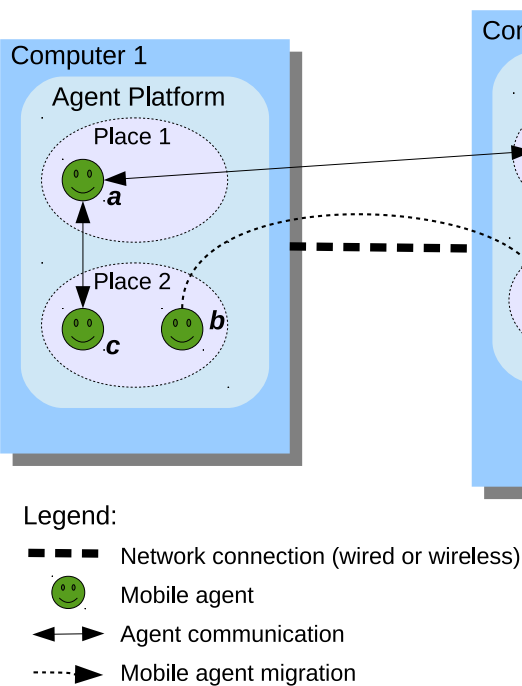

Fig. 2. Different elements in a mobile agent architecture.

vice to another by using a network connection. That is, while it is running on a certain computer or device it can pause its execution, transfer itself to another computer or device, and resume its execution in the new destination, as long as the transfer process ends successfully. For this to be possible, it is necessary that all the computers/devices involved (i.e., the origin computer/device and the target computer/device where the agent moves) execute a light middleware called the mobile agent platform [21] (e.g., Aglets, Voyager or SPRINGS/GeoSPRINGS [23,24], to cite some examples), which manages the movements of the agents and offers them a number of additional services [22] related to security, efficiency, communication, etc.

In Fig. 2, an example that summarizes the basics of a mobileagent based architecture is shown. There are two computers that can communicate by means of a network connection. Computer 1 executes a mobile agent platform with two places or execution environments for mobile agents, Place 1 and Place 2. Similarly, in Computer 2 there are two other places called Place 3 and Place 4. An agent executing in Place 1 (Agent a) establishes a communication with another agent (Agent $c$ ) that is executing in a different place inside the same platform (intra-platform communication), and also communicates with Agent $d$, that is placed in a different computer (Computer 2) using the network. Another agent (Agent $b$ ) is mobile and decides to change its execution environment, so it moves (by means of a network connection) to Place 4, which is hosted in a different computer (Computer 2), and then establishes a connection to a local database (which might be not accessible from a remote computer) to query data. Similarly, Agent $e$ is also mobile and performs a migration to another place inside the same computer (Place 3).

We think that mobile agent technology can provide relevant advantages for VANETs. Indeed, in a previous work, we have studied the potential use of mobile agents for data management in vehicular networks and analyzed the performance and benefits offered by that technology [8]. In these scenarios, the vehicles have computers capable of executing a mobile agent platform, as well as some wireless communication device. In this way, mobile agents can execute in such platforms and move from one vehicle to another as they travel, in order to process data in the distributed environment. As an example of application, mobile agents can be used to search and filter data that can be captured by different vehicles located along the streets of a city, using their sensors. The agents can reach spatial areas of interest by moving to them in two different ways, as shown in Fig. 3: through agent transmission, where

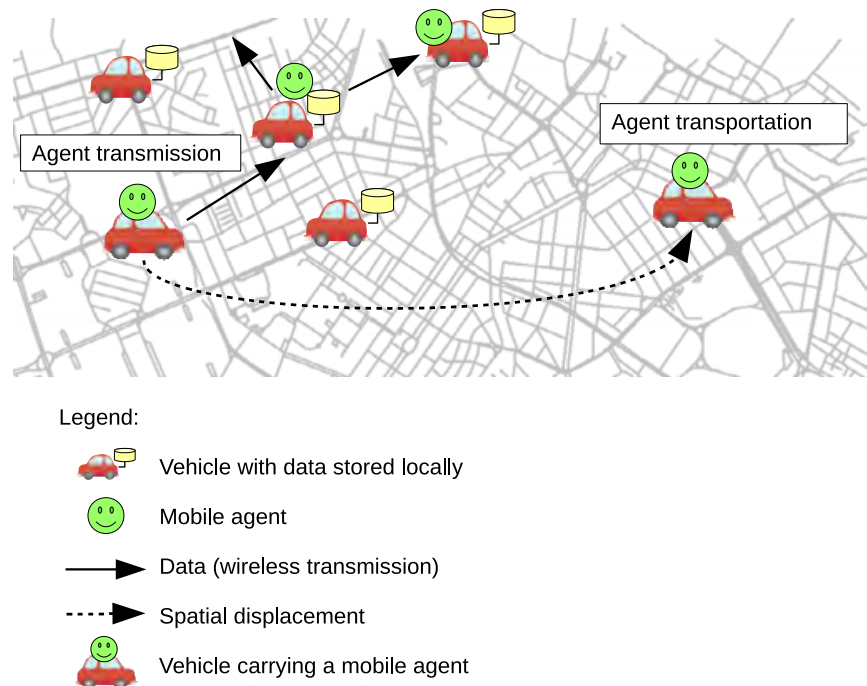

Fig. 3. Mobile agents in a VANET.

the mobile agent uses wireless communications to transfer itself from the execution environment located in an origin vehicle to another destination vehicle, and through agent transportation, where the mobile agent is physically transported to a different location by the vehicle that carries the computing device where the agent platform is being executed.

\section{Monitoring an interest area by using VANETs and mobile agents}

One proposed application for VANETs is the monitoring of certain parameters in an area by the moving cars present in it [25], and the possibility of executing queries about information related to that area (or any sub-area), that are processed by using the most updated data present in the VANET.

In this scenario, a percentage of equipped vehicles are assumed to carry a computer or device which collects data about its surroundings, that may be of interest for other drivers or even for users outside the roads. As an example of the first case, we could mention information about available parking spaces, the prices and status of gas stations or mechanical garages, the presence and occupation of recharging points for electric cars, the traffic density, etc. Besides that, it would also be very interesting if the vehicles carried different types of sensors to collect environmental information in the different places where the vehicle may travel to (e.g., to measure the ambient temperature, noise, solar radiation, or pollution gases such as $\mathrm{CO}_{2}, \mathrm{NO}_{2}, \mathrm{O}_{3}$, etc.).

In order to make this monitoring process more effective and the collected data more representative of the area, it is necessary to obtain readings from sensors in different places that, in addition, should be distant enough from each other. A simple way of achieving this is by dividing the interest area in portions or cells with the same surface following a grid layout. Then, samples can be taken once the involved sensors are located within each of those cells, which implies that if the sensors are carried by vehicles, they should visit all those places at some time, in order to obtain measured values from all the cells.

\subsection{Basic monitoring approach using mobile agents}

Traditional sensing approaches based on the use of stationary sensors and/or sensing stations can be expensive, which motivates the development of alternative or complementary solutions [26-28]. We argue that by using mobile agents in a VANET 
Table 1

Summary of advantages and disadvantages of several hop strategies described in [8].

\begin{tabular}{|c|c|c|c|}
\hline Hop strategy & Description & Advantages & Disadvantages \\
\hline EUC & Euclidean distance & Simple to compute & $\begin{array}{l}\text { It does not consider the street layout } \\
\text { The shortest distance is the only factor considered }\end{array}$ \\
\hline ANG & Frontal angle & It considers the vehicle's heading & $\begin{array}{l}\text { It does not consider the street layout } \\
\text { The direction of the vehicle is the only factor considered }\end{array}$ \\
\hline EP & Encounter probability & $\begin{array}{l}\text { It considers the vehicle's heading } \\
\text { It tries to estimate the probability that the vehicle will } \\
\text { reach the target area }\end{array}$ & It does not consider the street layout \\
\hline MAP & Street map distance & It considers the street layout & $\begin{array}{l}\text { It needs a digital map } \\
\text { It is computationally costly }\end{array}$ \\
\hline MapTraj & Trajectories using maps & It considers the vehicle's trajectories and the street layout & $\begin{array}{l}\text { It needs a digital map } \\
\text { The planned trajectories must be known }\end{array}$ \\
\hline Optimal & Optimal route & It returns the optimal sequence of hops & It is unrealistic (based on information that cannot be available) \\
\hline
\end{tabular}

it is possible to flexibly monitor potentially any geographic area required (interest area or target area) by using conventional vehicles and without the need to deploy a fixed sensor infrastructure. The whole monitoring process would consist of the following four steps:

1. Submission of the monitoring task. First, the user defines the parameters to be monitored within the bounds of a spatial area (e.g., the temperature in the city center during the afternoon), and immediately a mobile agent starts its execution with the defined monitoring parameters.

2. Travel to the interest area. In the second step, the mobile agent travels towards the interest area (in the example, the city center) by using the vehicles in the VANET to hop from one vehicle to another, using a specific hop strategy [8] (see Table 1 ), until it reaches the destination (transportation via wireless communications). Every time the mobile agent arrives at a vehicle, the next hop may not occur immediately, since the number of potential vehicles to hop to might be scarce (in the worst case, there might even be no other vehicles in the surroundings). In such cases, the mobile agent will stay in the same vehicle while waiting for a more suitable one. There is also the possibility of moving from one place to another by just staying in the current vehicle (transportation via locomotion). This combination of transportation facilities is sometimes called carry-and-forward or store-carry-and-forward in the context of vehicular networks [29].

3. Data collection. Once the mobile agent reaches the interest area, it will visit the different cells into which the area was partitioned. Upon its arrival to each of them, it will read the required sensor data and it will continue its travel to the next cell, repeating this process until all (or a preconfigured portion) of them have been visited. In order to reach each spatial cell in the grid, the mobile agent will likely need to hop from one vehicle to another in the same way as in the previous step. Notice that the vehicle where the agent is executing may move out of the interest area, and therefore in that case the mobile agent will need to find a way to come back to that area.

4. Return of the results. Finally, in the last step, the mobile agent returns the data collected in the interest area to its originator (e.g., a static node or a vehicle); again, ad hoc communications can be used to reach the place where the query originator is located. This stage of the process is challenging, as the originator could be a vehicle that may keep moving and thus constantly changing its spatial position. Thus, in this case, the mobile agent must estimate the position of the originating vehicle at every moment and, in case the estimation is wrong, keep re-evaluating and "chasing" the vehicle until it is successfully reached. The estimation of the position of the originating vehicle can be calculated according to its last known position and its intended destination (which will likely be the interest area). Such estimation will surely not be an exact position, but instead an area where the vehicle is expected to be present. The wider the area, the higher the existing uncertainty for the mobile agent to find the originator [8]; if needed, additional strategies could be used, for example, based on the use of vehicle-to-infrastructure communications through Road Side Units (RSUs) [4] or mailboxes to store the query results at fixed locations from which they can be easily retrieved [30].

One advantage of using mobile agents is that, instead of sending only data, they can carry along with them the logics or algorithms needed to acquire and filter out those data. Also, they can act as intelligent entities that can decide at every moment how to proceed according to the information they receive from their surroundings. This feature is especially useful in the scenario we are describing, in at least two aspects. Firstly, the mobile agent must reach the interest/target area by using the vehicles as intermediate nodes, being necessary to hop from one to another until the destination is reached. However, as explained before, one difficulty of VANETs is that their nodes move constantly, so it is not possible to use classic routing tables or compute the route to follow in advance; instead, the next node to be transferred to must be constantly evaluated just a few seconds before hopping to it, by using the information available to the mobile agent at that moment (its position and heading, the remaining distance to the target, etc.). Secondly, another advantage of carrying the logic with the mobile agent is that the agent can carry the specific algorithm needed to acquire and filter out the data involved in the monitoring process and thus minimize the amount of data transported (only the required data are carried). For example, the data acquisition task could be different if the monitoring is about the number of available parking spaces in a neighborhood than if it is about the average level of pollution gases in the area. In this way, instead of using a generic and potentially-heavy algorithm to acquire and filter out all the possible types of monitoring parameters, a more specialized and small code can be used for each case.

We believe that this is an interesting application for VANETs, that makes it possible to extract valuable information by using the vehicles as moving sensing platforms and the available sensors in a more efficient and dynamic way, if we compare it to the traditional solution based on static measuring stations deployed at fixed locations along fixed and static interest areas. Also, the use of mobile agents is adequate since their flexibility and autonomy makes them a suitable solution for a fast-changing scenario such as a VANET. The mobile agents encapsulate code to intelligently take decisions, based on available data about the environment, with the goal of overcoming difficulties appearing in a VANET environment, such as the limited time available for exchanging data (e.g., if there 
is not enough time to hop from one vehicle to another, due to the movements of the vehicles, the jump will fail and the mobile agent will retry or take an alternative decision). To reinforce this idea, we performed experiments to evaluate the basic monitoring approach described in this section, by using a mobile agent VANET simulator [31], which showed the feasibility of this proposal [8].

\subsection{Disadvantages of the basic monitoring approach}

The previously-described monitoring process has some drawbacks. For example, if the traffic density is too low, the mobile agent may not find a path to reach the interest area in a reasonable time, or even it may not reach it at all. Similarly, if a vehicle visited by a mobile agent leaves the area, or just parks in an underground parking before the agent hops away, it could get trapped or lost and the monitoring process would be interrupted.

Two possible strategies to try to limit these drawbacks are the setting of a timeout and the use of agent's clones. With the first strategy, once the mobile agent with the required monitoring parameters is launched, a timeout is set, and if the agent does not return any result before the time limit expires, the agent is assumed to be lost and launched again. In the second strategy, the mobile agent creates a number of copies of itself to increase the probability of successfully reaching the interest area by using different alternative routes [8]. This strategy increases the use of the network bandwidth, but maximizing the likelihood of obtaining a result can compensate this cost.

A potential crowdsourcing strategy could be that certain drivers (e.g., commuters) could make available the route that they will follow, to help the mobile agent to decide if a vehicle is worth to hop to or not. For privacy reasons, the complete routes are not expected to be provided with all the details. Indeed, this strategy does not necessarily require a full disclosure of the travel intentions of the driver. In general, only a minimum amount of information is needed, for an agent to know if a certain street is in the intended route or not. In exchange of this information, the driver can be compensated with virtual money (e.g., discount points for gas stations, virtual coins that can be used to pay for other useful information or even be exchanged by real money, etc.). This virtual money could be equal to 0 in the case of altruistic drivers that share their routes without requiring a profit, or in the case of public transportation vehicles that follow well-known and regular routes (such as bus or tramway lines).

Another strategy for enhancing the behavior of the monitoring process, which is the focus of this paper, is the use of spatial crowdsourcing techniques. For example, in a certain scenario, the mobile agent might find it difficult to reach certain areas of a city, but the user that starts the monitoring process may be willing to pay a certain amount of virtual money for some help from other vehicles. So, the mobile agent gets the ability to negotiate, with the drivers in the VANET, a way to reach the interest area faster or straighter than in the usual way. Recalling the steps followed to complete the monitoring process (described in Section 3.1), when the mobile agent hops to a vehicle in the second step of the process, it would not only look for other vehicles that seem to travel towards the interest area, but may also try to negotiate to be physically carried there in exchange of a certain amount of virtual money.

\subsection{Approach based on the use of spatial crowdsourcing}

As defined in [32], spatial crowdsourcing implies "locationspecific tasks that require people to physically be at specific locations to complete them". With spatial crowdsourcing, a vehicle could be willing to physically transport a mobile agent closer to

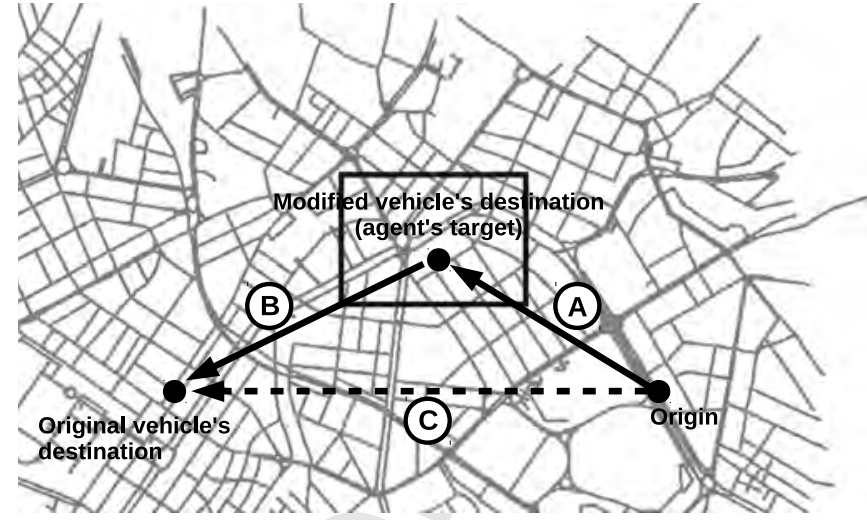

Fig. 4. Example of social cost calculation for a single vehicle.

its destination; we call such a vehicle a collaborator or collaborating vehicle. In this section, we describe our approach. Firstly, in Section 3.3.1, we introduce the concept of social cost. Secondly, in Section 3.3.2, we present the role played by virtual money in the spatial crowdsourcing approach. Thirdly, in Section 3.3.3, we present the basic workflow that describes the spatial crowdsourcing approach applied to travel to a target area. Finally, in Section 3.3.4, we describe the use of spatial crowdsourcing for data collection.

\subsubsection{Social cost}

Spatial crowdsourcing comes at a cost, as it usually requires an effort by the collaborating vehicles. Specifically, we define the social cost as the time needed by the collaborating vehicles to deviate from their original routes to carry the mobile agent towards its destination and, once the agent leaves the vehicle, to recover their previous destinations and continue traveling towards there. In other words, the social cost is the time that collaborators need to invest to help the mobile agent. More specifically, as shown in Fig. 4, the social cost is computed as the time needed by the collaborator to reach the agent's destination (A), plus the time needed to travel back to the driver's original destination (B), minus the time that it would have taken to directly follow the original route (C). If the agent would have not required this help, the collaborator would have not spent extra time traveling, so this cost needs to be compensated somehow.

\subsubsection{Virtual money}

Specifically, to encourage the collaboration from other vehicles, we propose an approach where a mobile agent can pay virtual money to them in exchange of being transported closer to the target area: the agent has a specific budget to process the query and tries to use that budget in the best possible way to reach its objectives and at the same time minimize the amount of money spent. The drivers of the collaborating vehicles could later use the virtual money received to pay other vehicles for similar services. The negotiation required to establish if a driver is willing to alter his/her current trajectory to follow the agent's objectives could be performed automatically whenever the agent is looking for the next vehicle to hop to; in order to accomplish this, the drivers of the area would state previously if they would like to participate in these exchanges and to what extent they are willing to detour from their route to bring the mobile agent closer to its destination. As an alternative, the driver could be explicitly asked if he/she is willing to follow a specified detour.

Several payment schemes could be considered. In our prototype, the amount paid by an agent is directly proportional to the time spent by the vehicle carrying the agent and there is no minimum fare, as shown in Table 2. The agent is assumed to have 
Payment model: payment from an agent to a collaborator.

Vehicle where the mobile agent is created ( $\left.V_{\text {origin }}\right)$ Mobile agent

Vehicle transporting the agent during $t$ time units

$\begin{array}{ll}\text { Initial budget } & \text { Final budget } \\ C_{V_{\text {origin }}}\left(\leq C_{V_{\text {origin }}}\right) & C_{V_{\text {origin }}}-C_{\text {agent }} \\ C_{\text {agent }}( & C_{\text {agent }}-t \\ C_{V_{\text {collaborator }}} & C_{V_{\text {collaborator }}}+t\end{array}$

an allowable budget (provided by the vehicle where the agent is created, that is, the monitoring node that wants to retrieve data from the interest area) and the agent will pay one unit of virtual money per every time unit (i.e., every second) spent in the vehicle acting as a "taxi" (i.e., following the route required by the agent). This route will likely be different than the one the vehicle was following originally. However, it might also happen that a driver obtains virtual money even if he/she does not really need to modify his/her trajectory to transport the agent (because the route required by the agent already matches the future trajectory of the vehicle) or that he/she gets a considerable amount of money in exchange of a slight deviation from the previous trajectory. This is unavoidable, as the intended trajectories of the drivers are unknown to the agent, and so the agent cannot know that it could achieve its objectives just by staying in the vehicle, even without paying for the vehicle to change its trajectory. Even though the basic payment scheme proposed is only based on transportation time, other additional costs could be easily added, if needed (e.g., the cost of traversing road tolls in case of interurban trajectories).

\subsubsection{Basic workflow of the spatial crowdsourcing approach}

The basic workflow of the proposed spatial crowdsourcing approach is shown in Fig. 5, where $S C$ is used as an abbreviation of spatial crowdsourcing. The following are the main steps of the process:

- In order to save virtual money, the mobile agent will first try to reach the interest area by hopping from one vehicle to another without asking vehicles to collaborate by modifying their original routes. Only if the speed at which the mobile agent approaches its destination (approaching speed) is lower than a certain value (1), which we call the minimum speed threshold, the agent will look for nearby collaborating vehicles (2) and will negotiate with them if they are willing to carry the agent. More specifically, the approaching speed of a mobile agent (ag) to the target area at time instant $t$ is defined as indicated in Equation (1), where $d(a g$, area, $t)$ represents the distance between the agent ( $a g$ ) and the target area (area) at time $t$, and $d(a g$, area, 0$)$ represents the initial distance between the agent and the target area; for simplicity of computation, we consider the Euclidean distance, but alternative distance metrics could also be considered. It should be noted that this approaching speed may not be the same as the speed at which the agent is traveling. For example, the agent may be in a vehicle moving at $50 \mathrm{~km} / \mathrm{h}$, but if its trajectory is inside a street located parallel to the target area then the approaching speed would be 0 , as that trajectory does not bring the agent closer to the target area. Similarly, if the mobile agent is traveling in the opposite direction to the target area, its approaching speed will have a negative value.

ApproachingSpeed $(a g, t)= \begin{cases}\frac{d(a g, \text { area }, t)-d(\text { agent }, \text { area }, 0)}{t}, & \text { if } t>0 \\ 0, & \text { otherwise }\end{cases}$

The minimum speed threshold must be either a positive value or 0 ; a value of 0 for the minimum speed threshold means

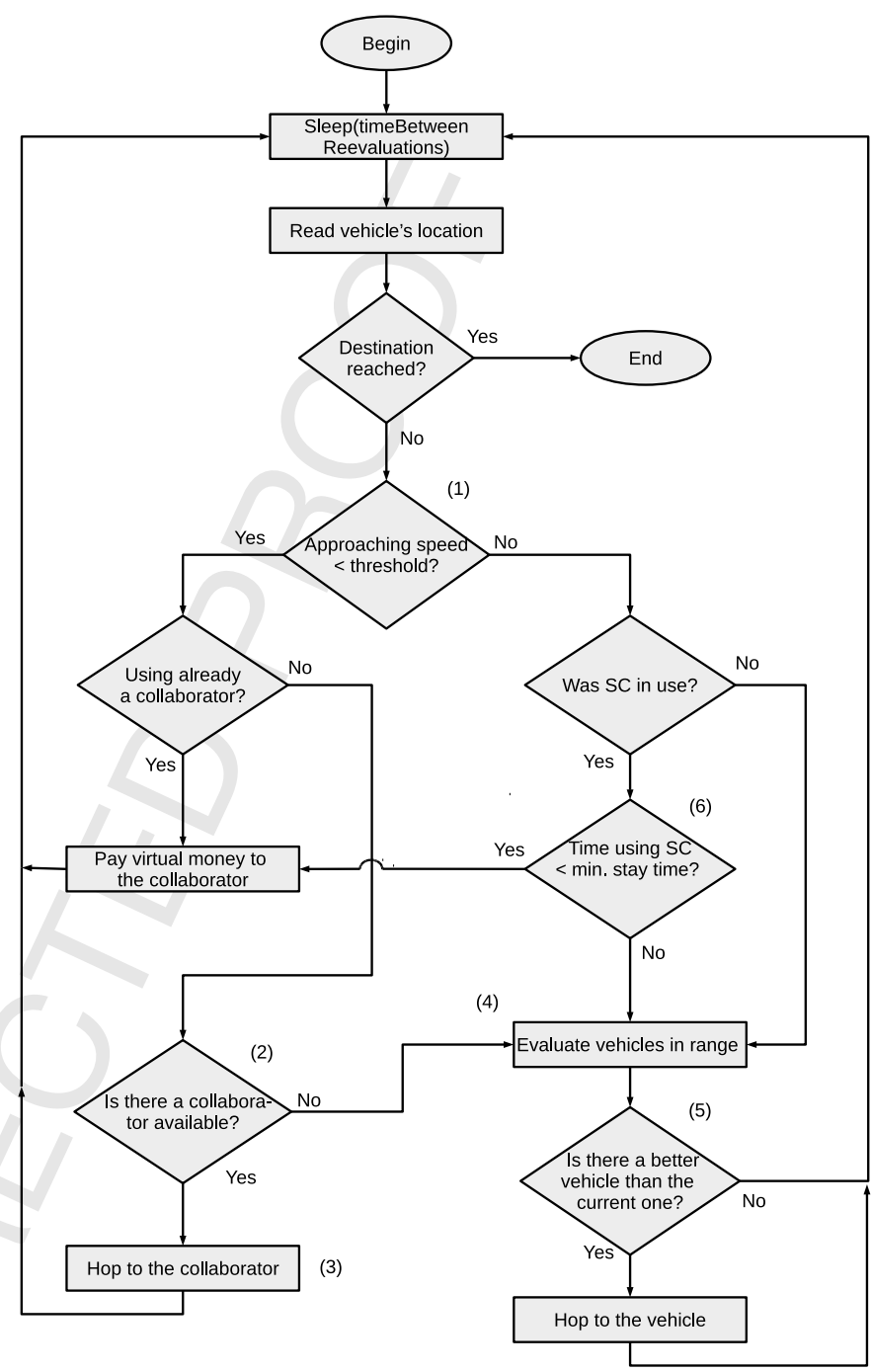

Fig. 5. Basic workflow of the SC approach followed by a mobile agent to travel to an area.

that the agent will never ask for help to potential collaborating vehicles (i.e., no spatial crowdsourcing will be used).

- If the potential collaborator accepts (3), then the mobile agent will stay in the vehicle and will not hop off it unless the approaching speed exceeds again the threshold value.

- If the driver does not agree to deviate from his/her trajectory (4), then the mobile agent will continue trying to reach the destination with the usual (non spatial crowdsourcing) approach, by hopping from one vehicle to another (5), while at the same time it keeps looking for a potential collaborator.

- If the mobile agent is already using a collaborator but it finds a suitable vehicle nearby, it can jump there (6). However, a minimum stay time in the vehicles can be considered for the following reason: for low values of the minimum speed threshold, once moving by staying in a collaborator it is easy to reach the threshold, and thus the agent may immediately hop to a more promising vehicle (i.e., one that approaches the target 
Table 3

Experimental settings.

\begin{tabular}{ll}
\hline Parameter & Default value \\
\hline City maps & Madrid, Barcelona, and Zaragoza \\
Size of the interest area & $0.25 \mathrm{~km}^{2}$ \\
Distance to the interest area & $2 \mathrm{~km}$ \\
Density of vehicles & Medium (100 vehicles/ $\mathrm{km}^{2}$ ) \\
Speed of the vehicles & $50 \mathrm{~km} / \mathrm{h} \pm 10 \%$ (random variability) \\
Mobility model & Pathway mobility model \\
Hop strategy & MAP (map distance) \\
Data collection delay & $5 \mathrm{~s}$ \\
Data to collect (number of samples) & 6 (1 from each cell of a $3 \times 2$ grid) \\
Data collection timeout & $3 \mathrm{minutes}$ \\
Total agent size (code, status, and amount of data carried by the agent) & $200 \mathrm{~KB}$ \\
Communication range & $250 \mathrm{~m}$ \\
Mobile agent's hop delay & $1 \mathrm{~s}$ \\
Minimum approaching speed threshold & $5 \mathrm{~km} / \mathrm{h}$ \\
Strategy used for data collection with SC & $\mathrm{SCCA}$ \\
Buildings block communication signals & Yes \\
Percentage of potential collaborators & $50 \%$ \\
Minimum stay time in a collaborating vehicle & $0 \mathrm{~s}$ \\
Size of the cold area (low-traffic area around the interest area) & $0 \mathrm{~m}$ \\
Percentage of trajectories crossing the cold area & $15 \%$ \\
Time limit & $20 \mathrm{minutes}$ \\
\hline
\end{tabular}

area faster). This may render the behavior of the mobile agent somewhat unstable, and in addition it may require a higher use of bandwidth, since the agent could be constantly hopping to new vehicles. So, in order to limit this effect, a hysteresis value for the agent's stay time (minimum period that the mobile agent must stay in a collaborating vehicle before hopping to others) is considered. In this way, the bandwidth used and the likelihood of switching continuously between spatial crowdsourcing and hopping among vehicles can be reduced. The impact of the value of this parameter is evaluated in Section 4.4.3.

So, in our proposal, we combine the basic monitoring approach, based on hopping from one vehicle to another by exploiting wireless communications, with spatial crowdsourcing. It should be noted that the switching between these two methods can occur several times at any moment during the trip to/from the target area. For example, if the agent is traveling too slowly (maybe because it is in a low-traffic street/area that makes it difficult to find other vehicles traveling towards the desired destination), it can use a collaborator to leave that street/area faster, and once its speed reaches a higher value, use again the method of hopping from one vehicle to another until the destination is reached.

It should be noted that we advocate a completely decentralized approach, based only on the use of opportunistic ad hoc communications with nearby vehicles. In this way, there is no need of a central entity with an overall view of the network in order to take global decisions and control the distribution of crowdsourcing tasks. Instead, the mobile agent itself takes its own decisions based on local knowledge about its environment and by interacting only with vehicles in its surroundings.

\subsubsection{Spatial crowdsourcing for data collection}

Spatial crowdsourcing could be used not only for traveling to the interest area but also for data collection once in the interest area. In order to know to which extent the spatial crowdsourcing is useful or not for the data gathering process, we have tested two versions of the mobile agent with different behaviors exhibited once the agent reaches the target area:

- In the first version, that we call SCCA (Spatial Crowdsourcing Collecting Agent), or SC/SCCA, the mobile agent always uses spatial crowdsourcing during the data collection phase (i.e., to travel to the target area cells and to return to the area if the vehicle leaves it during the data collection).

- In the second version, that we call PHCA (Pure Hopper Collecting Agent), or SC/PHCA, the mobile agent never uses the help of collaborators during the data collection phase; therefore, the agent tries to reach the cells within the target area by only hopping from one vehicle to another.

The difference between these two versions only affects phase 3 of the monitoring approach (see Section 3.1), since in both versions the mobile agent uses spatial crowdsourcing to travel to the area as well as to return the results to the originator vehicle. In Section 4.3, we show experimentally that the performance of the SCCA approach is better than one where spatial crowdsourcing is not used during the data collection phase.

\section{Experimental evaluation}

In this section, we present the experiments that we have performed to test the feasibility of the proposed monitoring approach in VANETs using mobile agents with spatial crowdsourcing abilities. For evaluation, we have used the MAVSIM simulator [31], that allows the simulation of both mobile agents and traffic in a realistic way by using real road maps extracted from OpenStreetMap [33]. Among its various functionalities, we can highlight the simulation of situations where the wireless signal is blocked by obstacles such as buildings, as this is more realistic.

\subsection{Experimental setup}

The main simulation parameters considered in the experiments carried out, with their default values, are shown in Table 3. In Section 4.1.1 we describe the main experimental parameters, and in Section 4.1.2 the metrics measured and the purpose of the different experiments performed.

\subsubsection{Main parameters in the simulations}

A few parameters included in Table 3 deserve further explanations:

- City maps. We have used map scenarios corresponding to different cities of Spain (Madrid, Barcelona and Zaragoza, as shown in Fig. 6), that exhibit different features regarding the presence of long straight road segments and short and/or 


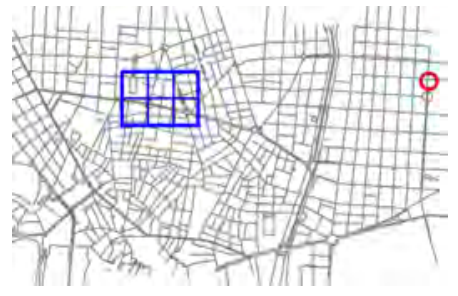

(a) Madrid

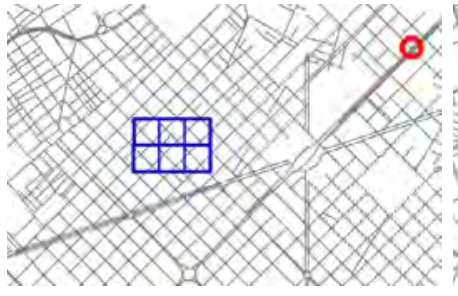

(b) Barcelona

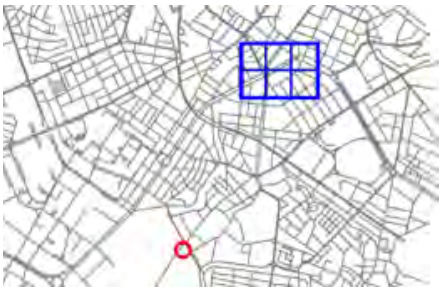

(c) Zaragoza

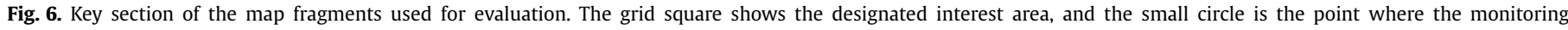
process is launched.

Table 4

Different metrics of the road graphs in the maps.

\begin{tabular}{llllllll}
\hline Map & $\begin{array}{l}\text { Length } \\
(\mathrm{km})\end{array}$ & $\begin{array}{l}\text { Surface } \\
\left(\mathrm{km}^{2}\right)\end{array}$ & $\begin{array}{l}\text { Road density } \\
\left(\mathrm{km} / \mathrm{km}^{2}\right)\end{array}$ & $\begin{array}{l}\text { Avg. edge } \\
\text { length }(\mathrm{m})\end{array}$ & $\begin{array}{l}\text { Vertices } \\
(\mathrm{V})\end{array}$ & $\begin{array}{l}\text { Edges } \\
(\mathrm{E})\end{array}$ & $\delta$ \\
\hline Madrid & 162.49 & 24.95 & 6.51 & 3.72 & 3732 & 4367 & 2.34 \\
Barcelona & 217.93 & 14.62 & 14.91 & 3.92 & 5060 & 5555 & 2.20 \\
Zaragoza & 253.3 & 18.76 & 13.50 & 3.54 & 6030 & 7153 & 2.37 \\
\hline
\end{tabular}

67

68

69

70

71

72

73

74

75

76

77

78

79

80

81

82

83

84

85

86

87

88

89

90

91

92

93

94

95

96

97

98

99

100

Fig. 7. The MAP hop strategy. map.

- Density of vehicles. The vehicle density can be defined as the number of vehicles present per surface unit [35] and it can be measured in terms of vehicles per square kilometer. Another similar vehicle density measure unit is the number of vehicles per road length unit [36], that is, vehicles per kilometer or per mile; this unit is useful for measuring the traffic flow in a mostly-linear road topology (such as a highway), but it is not suitable for urban scenarios, where vehicles can communicate not only with others present in the same street but also with vehicles traveling along other nearby parallel or perpendicular streets. Of course, this is not always possible due to the presence of obstacles such as buildings but, even in that case, opportunities can appear to perform the communication in open city areas such as main squares or street junctions. For these reasons, we use the vehicles per square kilometer (or vehicles $/ \mathrm{km}^{2}$ ) unit, since it is more appropriate for urban scenarios like those we are testing.

- Speed of the vehicles. The experiments are performed in an urban environment, so the speed of the vehicles is set to $50 \mathrm{~km} / \mathrm{h}$, which is a typical speed limit value in many countries for driving in a city. We introduce a random variability of $\pm 10 \%$ to that value, since it is highly unlikely that all the vehicles move exactly at the same speed. These settings are the same ones presented in [8].

- Hop strategy. The hop strategy refers to the algorithm used by the mobile agent when it travels by hopping among vehicles and it must choose, among several candidate vehicles, the one to hop to (instead of just being physically carried by a crowdsourcing collaborator). The agent needs to choose the vehicle that it estimates that will travel closer to the agent's destination with the highest probability. For the experimental evaluation, we have chosen by default a strategy that uses as a criterium the remaining distance to the destination (map distance), computed by adding the lengths of the streets to traverse in the road network. This algorithm is called the MAP hop strategy and an example is shown in Fig. 7: The mobile agent in vehicle $A$ travels towards its destination in $D$, and it must decide whether it hops to vehicle B or C; both vehicles have a similar straight (i.e., Euclidean) distance to D, but if the routes are computed following the streets, the distance $d 1$ from $\mathrm{B}$ to $\mathrm{D}$ is longer than the distance $d 2$ from $\mathrm{C}$ to $\mathrm{D}$; therefore, the mobile agent will choose the vehicle $C$, since it is the one with the shorter route to the destination. This hop strategy has been shown to provide good results [8].

- Mobile agent's hop delay. This is the time needed by the mobile agent to complete a hop from one vehicle to another, which implies transferring the agent's code, status and the data collected by the agent. We assume that in our test scenario the wireless technology used is the widespread $802.11 \mathrm{~g}$, that operates in ideal conditions at a maximum speed of 54 Mbps. However, a large number of factors can reduce the effective speed of the data transfer, such as the signal attenuation due to the distance and interferences and collisions with other devices operating in the area, as well as the processing overhead due to the packing and unpacking of the mobile agent's code and data (serialization and deserialization before and after transmission, respectively). Therefore, we do not assume a best-case scenario and establish the travel time to one second. Field experiments that we have performed with real smartphones and tablets confirm that this value is reasonable [8]. 
- Data to collect. Without loss of generality, in the experiments we assume that the interest area is partitioned based on a $3 \times$ 2 grid, which makes a total of six cells, which the mobile agent will have to visit to recover data from them.

- Data collection delay. When the mobile agent arrives in a spatial cell, it reads the corresponding vehicle's sensor, processes the value read, and stores it (if needed), and this action is assumed to take 5 seconds, which is a pessimistic estimation. During this interval, the mobile agent will not hop to any other vehicle until the sensor reading, processing, and storage, is complete. Therefore, the mobile agent not only spends time reaching the interest area and returning from it, but also reading, processing and storing values measured by sensors in the different cells.

- Data collection timeout. To avoid a potential situation where the data collection task takes too long, a timeout of 180 seconds is set. Reaching this timeout will cause the mobile agent to finish the task and start the process that intends to return the data to the originator vehicle or node where the monitoring process started. In this case, it might happen that the amount of data collected by the agent is smaller than the desired quantity. Therefore, the amount of collected data is a performance metric to consider.

- Time limit. To avoid situations where the monitoring process takes too much time to complete (for example, if an agent finds an unusually hard-to-solve situation), an overall time limit of 20 minutes is set. If this limit is reached, the simulation is aborted and its results are ignored, but the failure is counted and used to measure the reliability of the algorithm under the conditions that were being simulated. Thus, the time limit represents the maximum overall time that the user is willing to wait to obtain an answer.

In the experiments presented throughout this section, we use the default values shown in Table 3, using the three city maps, and vary one of the parameters, which will label the X-axis of the corresponding experimental figures. Thus, in Section 4.2.1 we vary the size of the cold area, in Section 4.2.2 the initial distance to the interest area, in Section 4.2.3 and Section 4.4.2 the density of vehicles, in Section 4.3 the minimum approaching speed threshold, in Section 4.4.1 the percentage of potential collaborators, and in Section 4.4.3 the minimum stay time in a collaborator. With this setup, we repeat every simulation 50 times (as in [8]), with different random starting positions for the vehicles, and compute the average of the results obtained.

\subsubsection{Metrics evaluated and purpose of the experiments}

The main metrics considered for evaluation purposes are the following:

- Time. An obvious metric to evaluate is the amount of time needed by the mobile agent to reach the interest area and/or perform the monitoring task.

- Amount of collected data. This metric indicates the number of cells in the interest area that the mobile agent visits and reads data from. In our settings there are 6 cells in the interest area, as we divided the interest area in a $3 \times 2$ grid, so we count the number of such cells that the mobile agent visits within the timeout of 3 minutes established for the data gathering phase. If this timeout is reached, then the mobile agent stops the data collection task and returns to the origin point with the data collected until that moment.

- Number of hops (bandwidth use). This metric counts the number of times that the mobile agent transfers itself from one vehicle to another one by using the wireless connection (i.e., the number of hops performed by the agent), and therefore it can be used as a measure of the bandwidth used by the whole process.

- Virtual money spent. This metric represents the payments made by the mobile agent in exchange of help from the collaborating vehicles.

- Social cost. As defined in Section 3.3, the social cost represents the extra time invested by collaborating vehicles in helping the mobile agent to perform its task, as compared to a situation where no help is provided (i.e., all the vehicles follow their intended routes).

With the payment scheme considered in this paper (where the agent pays in proportion to the stay time, as explained in Section 3.3), we expect a quite direct relation between the social cost experienced by the collaborating vehicles and the virtual money spent by the mobile agent and paid to the collaborating vehicles as a compensation. This means that the experimental figures measuring both metrics are expected to show similar trends. Nevertheless, notice that the relation cannot be computed as a direct proportion, as the social cost does not depend on the time spent by the collaborating vehicle carrying the agent but on the extra time needed to reach its intended destination despite the route deviation performed to help the agent. With other payment schemes (e.g., if the agent needs to pay a minimum fare every time it takes a collaborating vehicle), the relation between these two metrics (social cost and virtual money spent) can become blurred. Besides, it might happen that the collaborating vehicle's final trajectory may actually be very similar to the initially-intended one, as explained in Section 3.3: in this case, the virtual money spent by the agent may be higher than the social cost actually incurred by the collaborating vehicle.

- Reliability. The reliability of the monitoring is defined as the percentage of simulations that ended successfully within the simulation time limit established. This metric is very important as a complement of all the others. The reason is that all the previous metrics are computed by considering only the results of the successful simulations. Indeed, the metrics for unended simulations are not available, as the corresponding experiment did not end. It should be noted that the alternative of computing partial metrics or default metrics for unended simulations would be confusing; for example, considering a $0 \%$ of collected data for an unended simulation is misleading (in that case, it is inaccurate to say that the mobile agent collected $0 \%$ of the data; e.g., given just a few more seconds of simulation the simulation might have ended and the agent might have returned a good percentage of collected data); as another example, the number of hops so far in an unended simulation might be low but this might be only due to the fact that the simulation has not ended (given enough time for the simulation to end, the number of hops may actually turn out to be high).

To evaluate those metrics we performed two sets of experiments, whose purpose and conclusions are summarized in Table 5. The first set of experiments, shown in Section 4.2, is devoted to comparing the proposed spatial crowdsourcing approach with an approach without spatial crowdsourcing to travel to the interest area. The second set of experiments, described in Section 4.3, focus on determining a suitable value for the minimum approaching speed threshold. The third set of experiments, shown in Section 4.4, evaluates the impact of several parameters, such as the density of vehicles and the number of available collaborators, on the monitoring process. 
Table 5

Summary of the experiments performed and their purpose.

\begin{tabular}{|c|c|c|c|c|}
\hline Experiment & Section & Parameter & Purpose & Conclusions \\
\hline $\begin{array}{l}\text { Experiments Showing the } \\
\text { Interest of Spatial } \\
\text { Crowdsourcing to Travel to the } \\
\text { Interest Area }\end{array}$ & 4.2 & & $\begin{array}{l}\text { Evaluate the potential benefits of } \\
\text { spatial crowdsourcing (SC) to help a } \\
\text { mobile agent to reach an interest } \\
\text { area. }\end{array}$ & $\begin{array}{l}\text { The use of SC can help the mobile agent particularly } \\
\text { in low-traffic scenarios. Besides, even in scenarios } \\
\text { with good traffic density, the use of SC does not } \\
\text { have a negative impact. }\end{array}$ \\
\hline $\begin{array}{l}\text { Impact of the Size of a } \\
\text { Low-Traffic Area }\end{array}$ & 4.2 .1 & Size of the cold area & $\begin{array}{l}\text { Evaluate the impact of the size of a } \\
\text { cold area when SC is used or not. }\end{array}$ & $\begin{array}{l}\text { The use of SC is particularly beneficial in terms of } \\
\text { time and bandwidth for large cold areas. }\end{array}$ \\
\hline $\begin{array}{l}\text { Comparison Varying the Initial } \\
\text { Distance to the Interest Area }\end{array}$ & 4.2 .2 & $\begin{array}{l}\text { Initial distance to the } \\
\text { interest area }\end{array}$ & $\begin{array}{l}\text { Evaluate the impact of the initial } \\
\text { distance to the interest area when } \\
\text { SC is used or not. }\end{array}$ & $\begin{array}{l}\text { The use of SC is particularly beneficial for short and } \\
\text { medium distances to the target area. The bandwidth } \\
\text { usage obviously increases with the distance to the } \\
\text { interest area, but the use of SC minimizes it. }\end{array}$ \\
\hline $\begin{array}{l}\text { Comparison Varying the Density } \\
\text { of Vehicles }\end{array}$ & 4.2 .3 & Density of vehicles & $\begin{array}{l}\text { Evaluate the impact of the vehicle } \\
\text { density when SC is used or not. }\end{array}$ & $\begin{array}{l}\text { The use of SC is very beneficial when the density of } \\
\text { vehicles is low. }\end{array}$ \\
\hline $\begin{array}{l}\text { Experiments for the } \\
\text { Determination of a Suitable } \\
\text { Minimum Approaching Speed } \\
\text { Threshold }\end{array}$ & 4.3 & $\begin{array}{l}\text { Minimum approaching } \\
\text { speed threshold }\end{array}$ & $\begin{array}{l}\text { Evaluate the impact of the minimum } \\
\text { approaching speed threshold, and } \\
\text { choose the best value. }\end{array}$ & $\begin{array}{l}\text { A value of the approaching speed threshold as low } \\
\text { as } 5 \mathrm{~km} / \mathrm{h} \text {, to determine whether SC should be used } \\
\text { or not, provides good results. For higher threshold } \\
\text { values, the performance is also better than when not } \\
\text { using SC. }\end{array}$ \\
\hline $\begin{array}{l}\text { Experiments Analyzing the } \\
\text { Impact of Different Factors on } \\
\text { the Monitoring Process }\end{array}$ & 4.4 & & $\begin{array}{l}\text { Analyze the impact of different } \\
\text { parameters on the performance of } \\
\text { the whole SC monitoring process. }\end{array}$ & $\begin{array}{l}\text { The use of SC can benefit the monitoring process, } \\
\text { but a number of parameters must be set properly in } \\
\text { order to maximize the performance. }\end{array}$ \\
\hline $\begin{array}{l}\text { Impact of the Percentage of } \\
\text { Potential Collaborating Vehicles }\end{array}$ & 4.4 .1 & $\begin{array}{l}\text { Percentage of potential } \\
\text { collaborators }\end{array}$ & $\begin{array}{l}\text { Evaluate the impact of the } \\
\text { percentage of collaborating vehicles. }\end{array}$ & $\begin{array}{l}\text { A low percentage of collaborators (about } 10 \% \text { ) is } \\
\text { enough to benefit from SC. }\end{array}$ \\
\hline Impact of the Density of Vehicles & 4.4 .2 & Density of vehicles & $\begin{array}{l}\text { Evaluate the impact of the density } \\
\text { of vehicles (both collaborating and } \\
\text { not collaborating) in the scenario. }\end{array}$ & $\begin{array}{l}\text { Even with a low traffic density ( } 60 \text { vehicles } / \mathrm{km}^{2} \text { ), } \\
\text { the use of SC provides good results. }\end{array}$ \\
\hline $\begin{array}{l}\text { Impact of the Minimum Stay } \\
\text { Time in a Collaborator }\end{array}$ & 4.4 .3 & $\begin{array}{l}\text { Minimum Stay Time in } \\
\text { Collaborators }\end{array}$ & $\begin{array}{l}\text { Evaluate the impact of the } \\
\text { minimum time that the mobile } \\
\text { agent stays in a collaborator. }\end{array}$ & $\begin{array}{l}\text { High staying times do not affect the total time or } \\
\text { the reliability, but increase both the social and } \\
\text { economic costs. }\end{array}$ \\
\hline
\end{tabular}




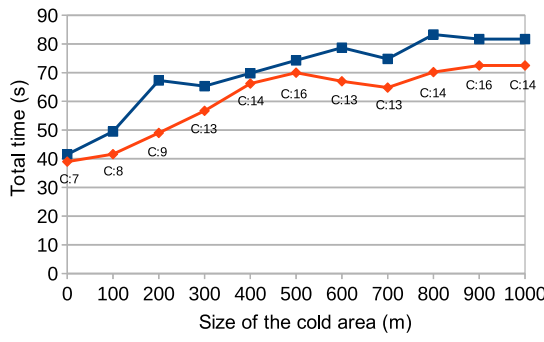

(a) Madrid

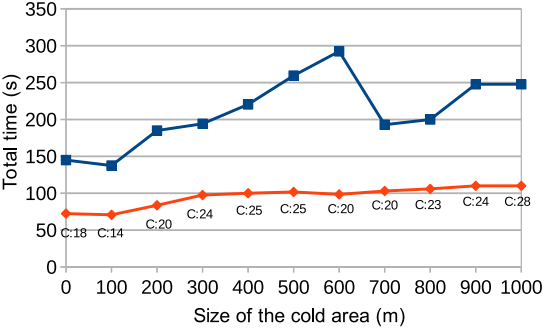

(b) Barcelona

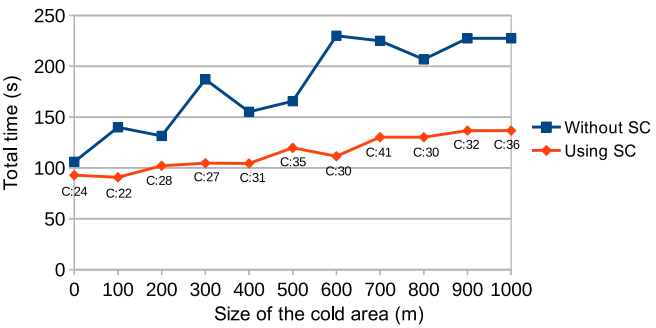

(c) Zaragoza

Fig. 9. Traveling to the interest area: comparison of the total time needed by using spatial crowdsourcing (SC) or not, varying the size of the cold area.

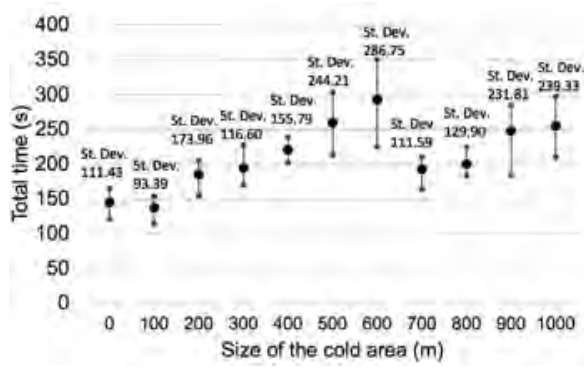

(a) Not using SC

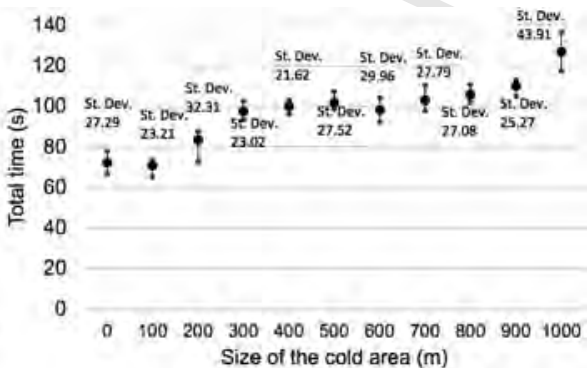

(b) Using SC

Fig. 10. Confidence intervals for Fig. 9(b) - city of Barcelona.

trajectories of the vehicles and the communications among them vary with the different topologies of the streets.

The difference in the total times shown in Fig. 9(b) for cold areas of size 600 and 700 meters when no spatial crowdsourcing is not used may attract the attention of the reader; this is because, in that case, the mobile agent finds more difficulties with a size of the cold area of 600 meters than with a size of the cold area of 700 meters, and therefore we observe a drop in the total time when the size of the cold area goes from 600 meters to 700 meters. Although this might seem counter-intuitive, as one may expect that a larger cold area would imply higher difficulties for the agent, we also have to take the topology of the streets into account. Indeed, it is not only the size of the cold area that matters but also the number and shape of the streets close to the boundaries of the cold area. So, by analyzing the results in detail, we have noticed that with a size of the cold area of 600 meters, in some simulations the agent tries to keep itself for a considerable amount of time in a narrow area close to the cold area until it finds a suitable vehicle to go towards the interest area, rather than exploring other alternatives, because it takes local decisions that lead to a local optimum (exploring other streets in those scenarios would initially lead the agent farther from the interest area). These problematic situations are avoided when the agent uses spatial crowdsourcing, as the agent would detect little progress towards the interest area (the approaching speed would be below the minimum approaching speed threshold) and ask the help of potential collaborating vehicles.

When spatial crowdsourcing is not used, we also observe a higher variability in the total times of different executions, as the approach with no spatial crowdsourcing is more sensitive to changes in the scenario. For illustration, in Fig. 10(a), we show the $95 \%$ confidence intervals of the average time values shown in Fig. 9(b) for the case without spatial crowdsourcing (the thicker circles represent the average values and the extremes the higher and lower values defining the interval), along with labels to indicate the standard deviations (St. Dev.) computed from the 50 executions for each value of the size of the cold area. Similarly, in Fig. 10(b), we show the confidence intervals and standard de- viations for the case of using spatial crowdsourcing. Notice the different scale in the Y-axis of Fig. 10(a) and Fig. 10(b).

In Fig. 11, the total number of hops is shown. When spatial crowdsourcing is used, the number of times that the mobile agent hops (and so the bandwidth needed) is smaller: when the agent finds a collaborating vehicle, it is directly carried towards the interest area and does not need to use any other vehicle to hop to, unless the agent's approaching speed decreases below the minimum speed threshold and there is some promising neighboring vehicle; in this experiment, this is unlikely because the carrying vehicle is usually moving through the cold area, which is a lowtraffic zone. On the contrary, when no collaborators are used, the mobile agent is constantly looking for a carrier better than the one it is currently traveling on. In this case, the current carrier may follow unpredictable routes, not only carrying the agent nearer the interest area; indeed, it might take a route that travels farther from the interest area. The size of the cold area seems to have little influence on the number of hops. There are two reasons for that. When using spatial crowdsourcing help, once a collaborator is found the mobile agent does not usually need to hop to other vehicles and stays in the same vehicle moving towards the interest area. When no spatial crowdsourcing is used, the number of hops varies with the size of the cold area in a more irregular way. This is due to the unpredictability of the routes and locations of the vehicles: the mobile agent must constantly evaluate its environment to decide the most suitable vehicle.

We also evaluated the social cost of using spatial crowdsourcing. In Fig. 12, we show the social cost (in minutes) in the different cities, when the mobile agent uses spatial crowdsourcing (when spatial crowdsourcing is not used, there is no social cost). Regarding the size of the cold area, it has little effect in the social cost, although a slight increase with its size is observed. This is an expected consequence, since the larger the cold area is, the longer it takes a collaborator to transport the agent to the interest area, and the longer he/she must postpone following his/her originallyintended route.

To summarize the conclusions regarding the influence of the size of the cold area, when it is larger the total time needed by 


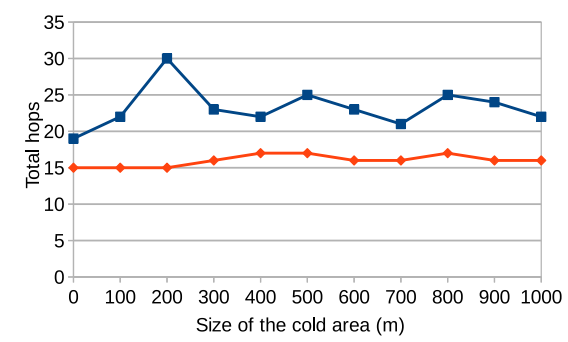

(a) Madrid

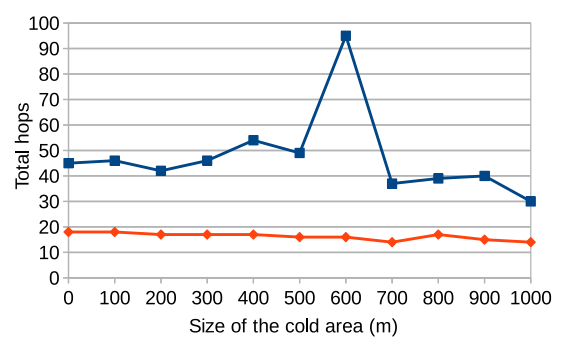

(b) Barcelona

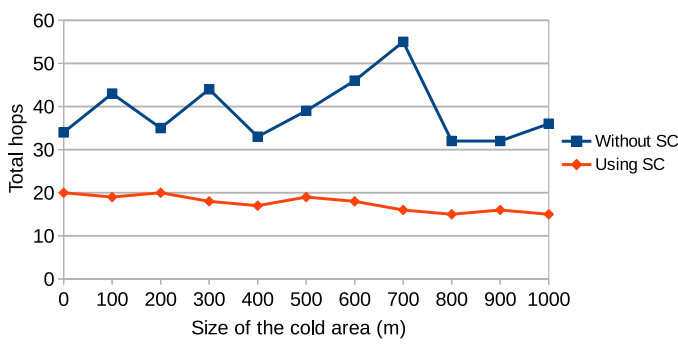

(c) Zaragoza

Fig. 11. Traveling to the interest area: comparison of the total number of hops needed by using spatial crowdsourcing (SC) or not, varying the size of the cold area.

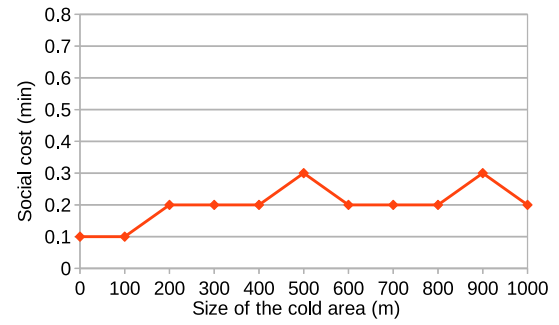

(a) Madrid

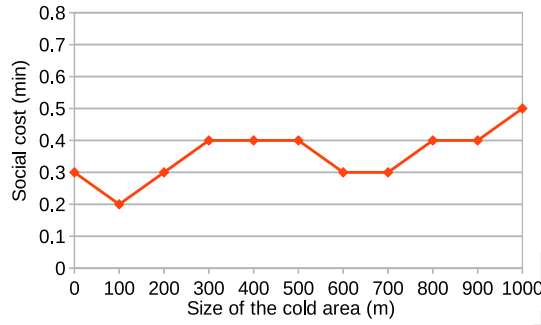

(b) Barcelona

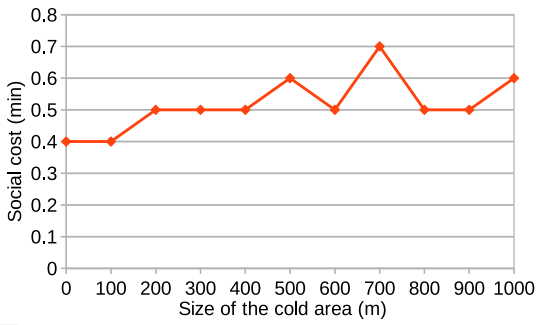

(c) Zaragoza

Fig. 12. Traveling to the interest area: collaborators' social cost.

the mobile agent to reach the interest area grows, although it is smaller when spatial crowdsourcing is used. The bandwidth usage remains similar, but when no spatial crowdsourcing is used it is higher and more irregular. Finally, the social cost, which only applies when using spatial crowdsourcing, is only slightly influenced by the size of the cold area.

\subsubsection{Comparison varying the initial distance to the interest area}

The previous experiment indicates that spatial crowdsourcing can be very useful in scenarios with areas where the density of vehicles is not high. Now, we will perform another experiment with higher traffic density in the whole scenario, in order to see if the proposed approach also behaves well when the number of potential agent carriers increases.

In this experiment, there is no cold area and there is a uniform density of vehicles in the scenario, which is set to 100 vehicles $/ \mathrm{km}^{2}$ (a medium density), as the default values in Table 3 indicate. We vary the initial distance from the point where the mobile agent starts its execution to the limit of the interest area, and we evaluate the potential benefits of spatial crowdsourcing for different values of such a parameter.

In Fig. 13, we can see how the total time to reach the interest area varies with the initial distance. In general, the times are slightly higher when no spatial crowdsourcing is used for the smaller to medium initial distances, whereas for higher distances the differences are near 0 and practically the same. A minor exception can be found in Fig. 13(c), for the city of Zaragoza, in a couple of cases where for some distance values (e.g., 1750 and 2250 meters) the times are slightly higher when spatial crowdsourcing is used, but this difference is quite small (about 3 seconds, from a total of around 67 seconds and 80 seconds, respectively); this difference is very small and can be due to a single sub-optimal choice made by the agent (e.g., a decision about being transported rather than actively jumping to a promising vehicle).

Regarding the number of hops that the mobile agent performs to reach the interest area, shown in Fig. 14, the figures for all the cities have a similar shape: the number of hops is slightly higher when no spatial crowdsourcing is used, and it grows linearly with the distance to the interest area.
As a summary, the time needed to reach the interest area grows with the distance. For short and medium distances, the spatial crowdsourcing option is better, but for longer distances the difference between both options is smaller. Regarding the total number of hops needed, the initial distance has a linear effect and in all the cities the crowdsourcing option takes fewer hops than the one without spatial crowdsourcing.

\subsubsection{Comparison varying the density of vehicles}

The density of vehicles is very important for the performance of the agent, since it can be difficult to reach the interest area if the number of vehicles that can be used as a physical transport or as an intermediate relay is low. In this experiment, we want to know in which circumstances the use of spatial crowdsourcing helps the mobile agent to reach the interest area. In this scenario, there is no cold area and in all the zone there is a uniform density of vehicles, which varies from 10 vehicles $/ \mathrm{km}^{2}$ (a very low density) to 175 vehicles $/ \mathrm{km}^{2}$ (a high density).

In Fig. 15, we can see how the total time to reach the interest area varies with the density of vehicles. In all the cities, the time is lower when spatial crowdsourcing is used than when it is not. The differences are higher with low vehicle density values, especially for the lowest value of 10 vehicles $/ \mathrm{km}^{2}$. For density values higher than 30 to 45 vehicles $/ \mathrm{km}^{2}$ the difference between using spatial crowdsourcing or not is practically negligible and in some cases (e.g., Fig. 15(c)) the total time might even increase very slightly when using spatial crowdsourcing. The reason is that, when the traffic density is high and in the absence of a cold area, the mobile agent can find suitable vehicles to hop to in order to transport itself via wireless communications. When spatial crowdsourcing is not used, the mobile agent must go to the interest area by hopping from one vehicle to another that seems more promising to reach the target sooner, and finding candidates is more difficult when the density of vehicles (and therefore, its total amount) is lower.

Fig. 16 shows that, as the vehicular density grows, the number of hops that the mobile agent needs to perform to reach the interest area decreases when spatial crowdsourcing is not used. On the other hand, when it is used, the number of hops increases with the density of traffic until around 45 vehicles $/ \mathrm{km}^{2}$, when the num- 


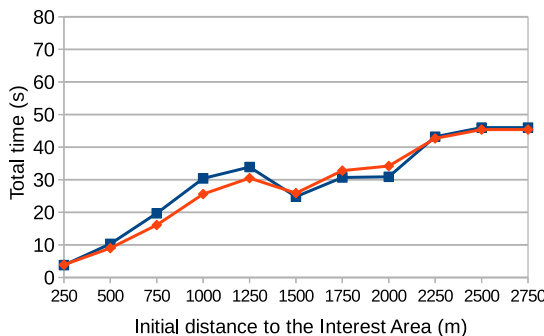

(a) Madrid

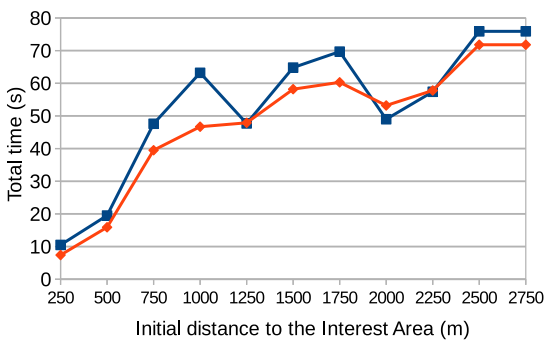

(b) Barcelona

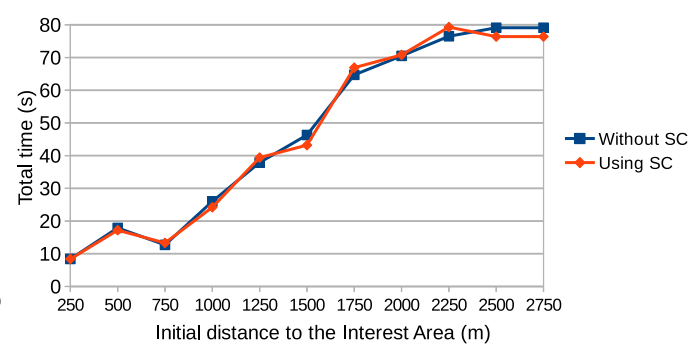

(c) Zaragoza

Fig. 13. Traveling to the interest area: comparison of the total time needed by using spatial crowdsourcing (SC) or not, varying the initial distance (no cold area).

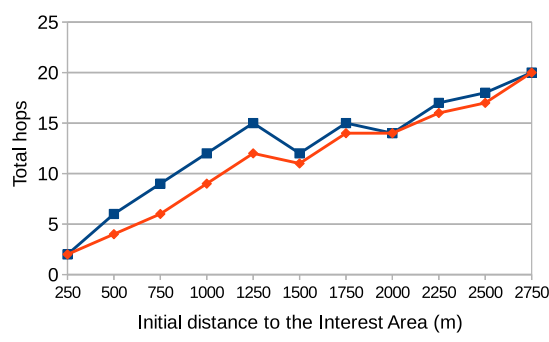

(a) Madrid

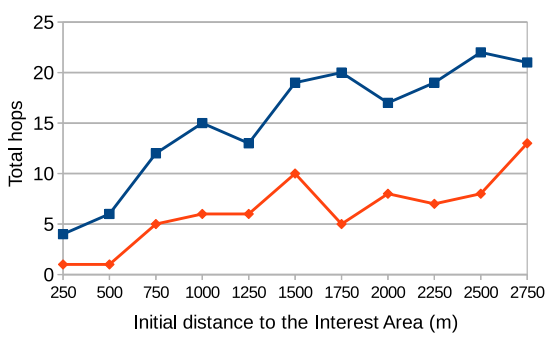

(b) Barcelona

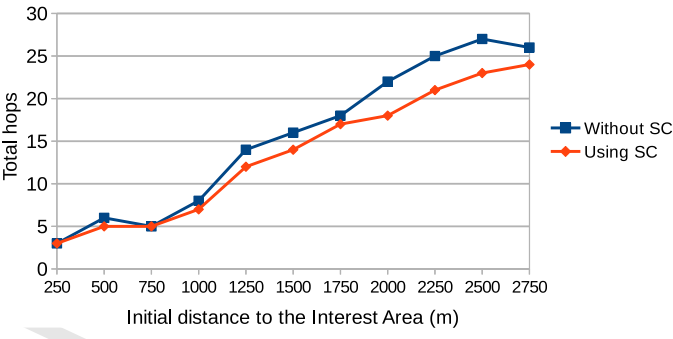

(c) Zaragoza

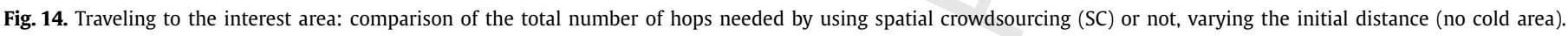

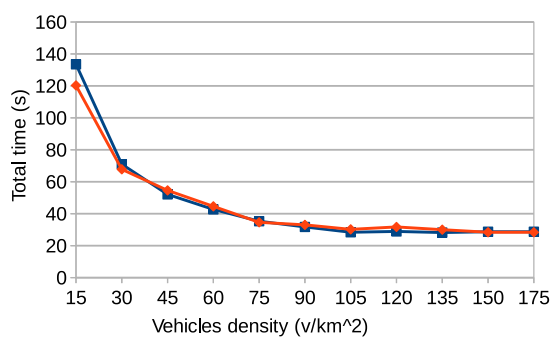

(a) Madrid

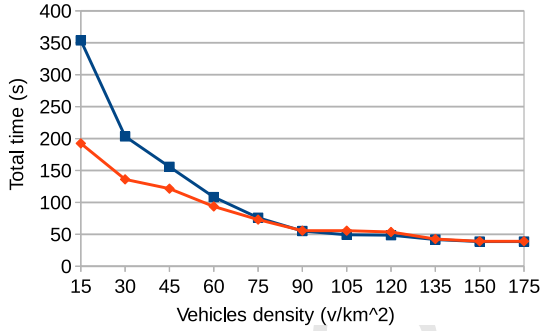

(b) Barcelona

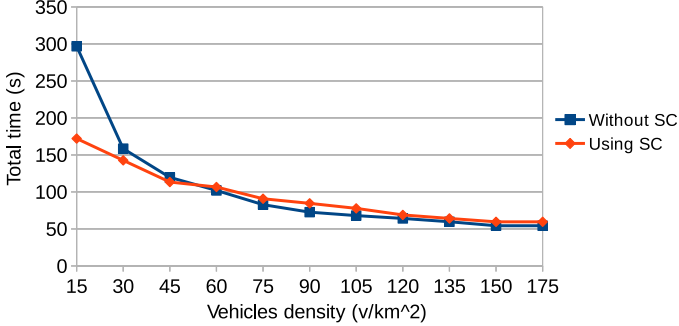

(c) Zaragoza

Fig. 15. Traveling to the interest area: comparison of the total time needed by using spatial crowdsourcing (SC) or not, varying the vehicle density (no cold area).

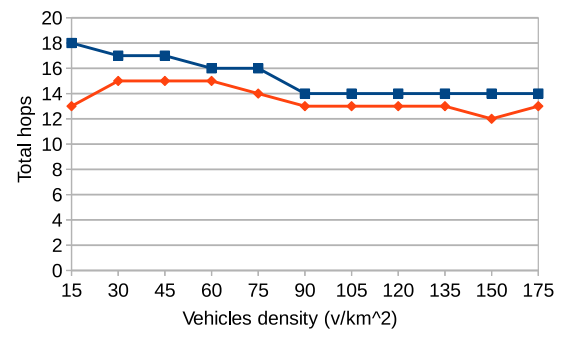

(a) Madrid

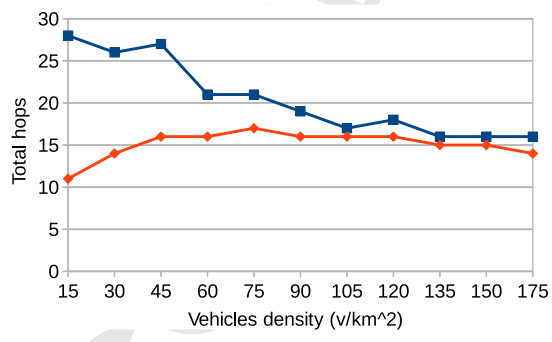

(b) Barcelona

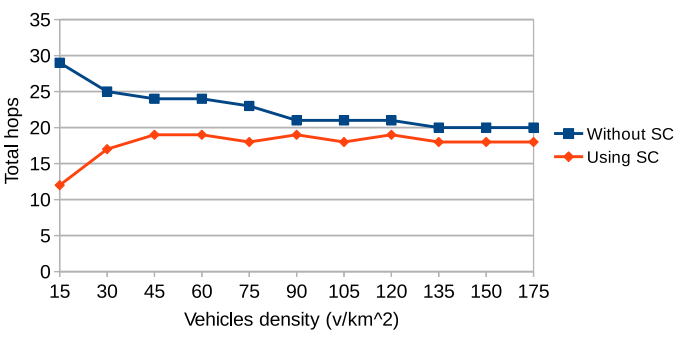

(c) Zaragoza

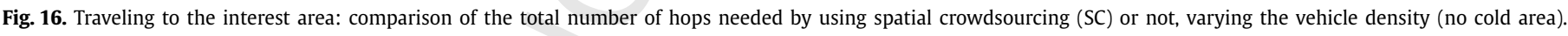

ber of hops remains stable with small variations for higher density values. In all the cases, the number of hops when spatial crowdsourcing is used is smaller than when it is not used, although the difference decreases as the vehicular density grows. This is due to the fact that, once the mobile agent takes a collaborator, it stays in there and does not leave it until the interest area is reached, being unnecessary to perform more hops to other vehicles.

Summing up, we conclude that the spatial crowdsourcing approach is particularly beneficial in terms of time and bandwidth usage in scenarios with a low density of vehicles. Moreover, when the traffic density is higher, the use of spatial crowdsourcing does not harm either the performance. Indeed, based on the minimum speed threshold, the mobile agent can choose to hop among the vehicles when it considers that paying a collaborator is not needed.

\subsection{Experiments for the determination of a suitable minimum approaching speed threshold}

In the second set of experiments, we focus on the determination of a suitable value for the minimum approaching speed threshold parameter used by a mobile agent to decide when collaborators should be used. As explained in Section 3.3, the travel to the target area, as well as the return to the origin with the results, is performed by the mobile agent thanks to a combination 
of two techniques: initially, the mobile agent starts hopping from one vehicle to another one that it considers to be a more promising carrier to reach the target area and, additionally, the agent may also use spatial crowdsourcing to be physically carried nearer the target area by a collaborating vehicle. To decide whether using one technique or the other at any time, a minimum speed threshold is set: if the mobile agent approaches the destination area with a speed smaller than this threshold value, it will ask for help to nearby collaborating vehicles to be carried nearer the destination area faster, until the approaching speed raises again above the threshold. Otherwise, the mobile agent will keep hopping from one vehicle to another, as described before. It is important to establish the minimum speed threshold carefully. If it is too high, the mobile agent will likely look for collaborators too early, and therefore it will spend too much virtual money needlessly. On the other hand, if the value is too low, then the agent will ask for help later, but then it may be already too far from the interest area and thus take too much time to reach the destination.

In Section 3.3.4, we describe two approaches regarding the use of spatial crowdsourcing during the data collection phase (SCCA and PHCA), which we now compare experimentally for varying values of the minimum approaching speed threshold (in the range from $0 \mathrm{~km} / \mathrm{h}$ to $50 \mathrm{~km} / \mathrm{h}$, which is the usual maximum speed within a city). In the rest of this section, the results obtained by using SCCA are shown in Section 4.3.1, the results obtained by using PHCA are shown in Section 4.3.2, and a comparison between the two is presented in Section 4.3.3. As the default parameter values in Table 3 show, the ratio of collaborating vehicles is set to $50 \%$, taken as an intermediate value, and there is no cold area. Besides, as we want to see the benefits of using spatial crowdsourcing also within the interest area, the probability that the trajectory of a given vehicle will travel inside the interest area is $10 \%$, as this implies a low-traffic situation where the difference between using spatial crowdsourcing or not using it during the data collection phase can be more relevant.

\subsubsection{Monitoring process: results with SCCA}

Fig. 17(a) shows the time required for completing the monitoring process in the three cities, by using the SCCA variant of the mobile agent, as described before. The extreme case when the minimum speed threshold equals $0 \mathrm{~km} / \mathrm{h}$ means that the condition for using spatial crowdsourcing is never met, and therefore the agent must reach the target area only by hopping from one vehicle to another (spatial crowdsourcing is not used at all). However, once the target area is reached, the SCCA mobile agent always uses spatial crowdsourcing to travel to all the cells, with independence of the minimum approaching speed threshold (the agent is already within the target area, and so the concept of approaching speed does not apply). As a result, in general, in all the maps, the total time required is higher with a value of $0 \mathrm{~km} / \mathrm{h}$ than with other higher values, and the variations for higher minimum speed thresholds are quite small.

In Fig. 17(b), we can observe that the number of hops needed by the mobile agent to complete the process is much higher for the minimum speed threshold value of $0 \mathrm{~km} / \mathrm{h}$ (when no spatial crowdsourcing is used), and for values higher than $5 \mathrm{~km} / \mathrm{h}$ the number of hops decreases. The reason is that when no spatial crowdsourcing is used the mobile agent needs to constantly hop from one vehicle to another until it reaches the interest area.

In Fig. 17(c), the percentage of collected data is shown. The result for all the minimum speed threshold values tested are $100 \%$ (i.e., all the requested data are collected), but it must be taken into account that for the value of $0 \mathrm{~km} / \mathrm{h}$ (i.e., when no spatial crowdsourcing is used) the reliability is below $90 \%$, so the collected data percentage shown in Fig. 17(c) refers only to the simulations ended within the simulation time limit.
In Fig. 17(d), the amount of virtual money spent by the mobile agent is shown. As explained in Section 3.3, in our experiments, we assume that the agent pays one unit of virtual money for every second it is carried by a collaborating vehicle. When the minimum speed threshold value is $0 \mathrm{~km} / \mathrm{h}$, the amount paid is very low, since the collaborators are only used when the mobile agent collects data when traveling to the target area cells within the interest area, but not for reaching the area when returning to it when the agent leaves it unintentionally, or for returning to the monitoring origin. For higher values of the minimum speed threshold, the virtual money spent increases, being the best overall value around $5 \mathrm{~km} / \mathrm{h}$

Regarding the social cost, shown in Fig. 17(e), the SCCA agent exhibit a similar behavior in the three cities: when the minimum speed threshold value is $0 \mathrm{~km} / \mathrm{h}$, the cost is very low because spatial crowdsourcing is used only when the mobile agent is inside the target area collecting data. For higher speed threshold values, the social cost also increases, since the agent makes use of spatial crowdsourcing more frequently. Note that this graphic shows a trend very similar to the one of the virtual money spent (though not exactly the same), which means that the established mechanism to compensate the collaborators (paying them according to their invested time) is quite fair, since the compensation paid is related to the social costs incurred by the collaborators.

Finally, Fig. 17(f) shows the number of collaborating vehicles that the SCCA agent needs to complete the process. For the minimum speed threshold value of $0 \mathrm{~km} / \mathrm{h}$, the number of collaborators is very low, because they are only used in the data collection phase, but not for traveling to/from the target area. For the next minimum speed threshold value $(5 \mathrm{~km} / \mathrm{h})$, the number of collaborators grows to between 11 and 15 , but then it decreases as the minimum speed threshold value increases, until 6 to 10 collaborators for $50 \mathrm{~km} / \mathrm{h}$. This is interesting, as compared to the results of the total time, which has a low variability for minimum approaching speed threshold values in the range of 5 to $50 \mathrm{~km} / \mathrm{h}$ (see Fig. 17(a)). When the minimum approaching speed is set to a low value (e.g., $5 \mathrm{~km} / \mathrm{h}$ ), the mobile agent uses more collaborators but for a shorter time. On the other hand, when the minimum approaching speed is set to high values (e.g., $45 \mathrm{~km} / \mathrm{h}$ ), fewer collaborators are used by the mobile agent, but it travels physically aboard them for a longer time. The reason is the following: when the agent uses spatial crowdsourcing it will stay in the collaborating vehicle until the approaching speed exceeds the minimum speed threshold, and shortly after that moment the agent will leave the collaborator if it finds a more promising vehicle, and will continue approaching the target area, hopping from one vehicle to another by using the established hop strategy. For low values of the minimum speed threshold, it will be easier to reach the threshold value, and the agent will leave the collaborator sooner than for high values. As the minimum approaching speed threshold grows, the mobile agent will travel longer in a collaborator, and will approach nearer the target area, so it will also need a smaller number of collaborators to reach it.

It should be noted that the performance results of unended simulations are not available, and so another parameter tested in the experiment is the reliability of the monitoring. In Table 6, we can observe that the reliability of the process is between $56 \%$ and $90 \%$ when then minimum speed threshold equals $0 \mathrm{~km} / \mathrm{h}$ (i.e., when no spatial crowdsourcing is used). For higher values of the minimum speed threshold, the reliability reaches $100 \%$ (with one single exception for the minimum speed threshold value of $40 \mathrm{~km} / \mathrm{h}$ and the city of Zaragoza, with a reliability of $98 \%$ ), meaning that the simulations end within the time limit. That is, the use of spatial crowdsourcing for values above or equal to $5 \mathrm{~km} / \mathrm{h}$ increases the reliability of the whole monitoring process considerably. 


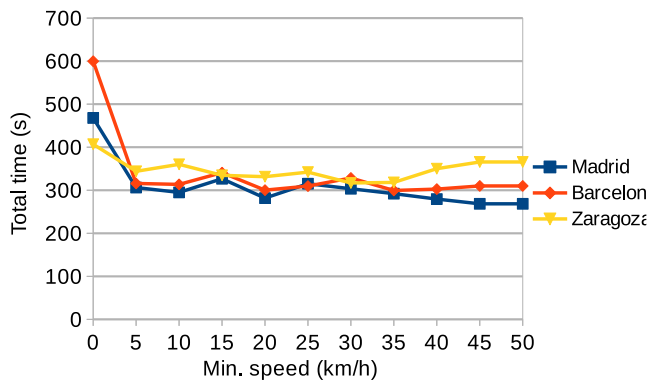

(a) Time

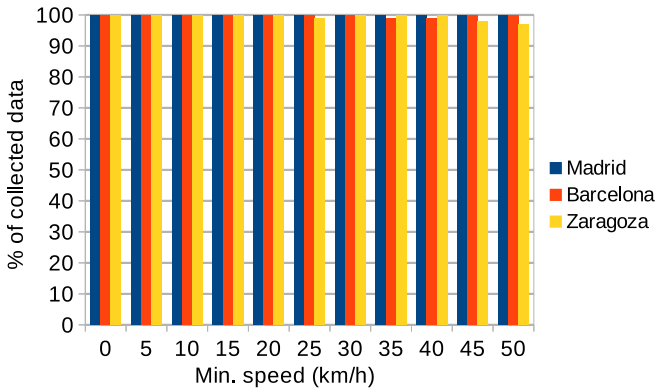

(c) Collected data

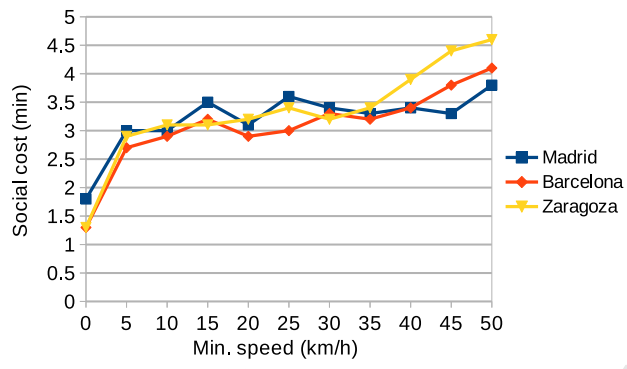

(e) Social cost

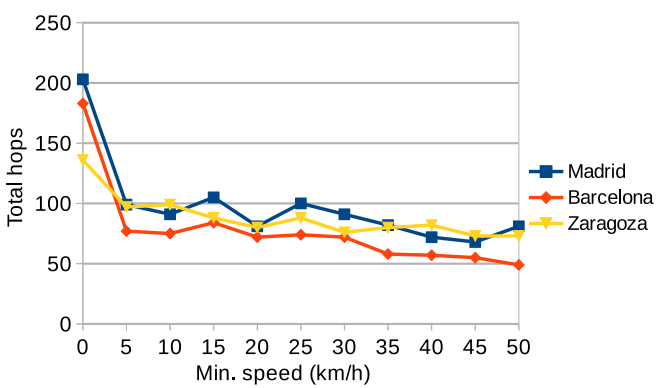

(b) Number of hops

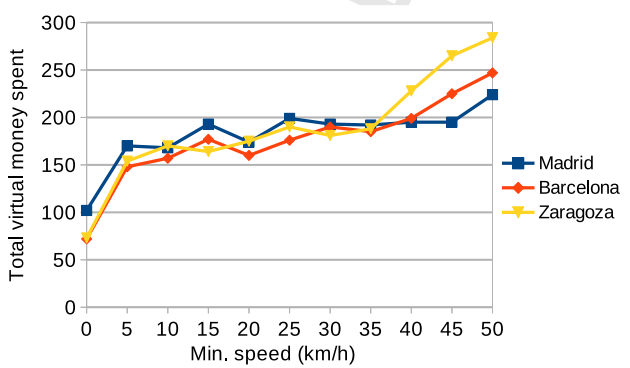

(d) Virtual money

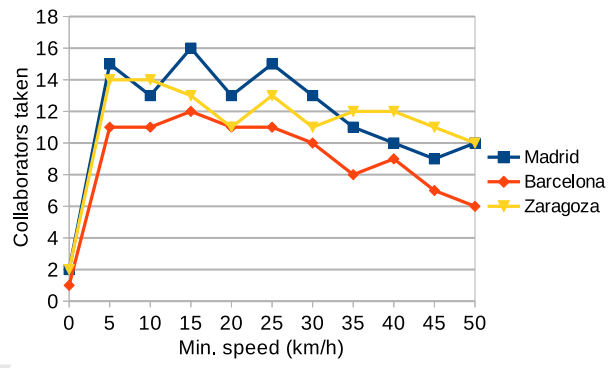

(f) Number of collaborators

Fig. 17. Monitoring process: results with SCCA.

Table 6

Monitoring process: reliability with SCCA.

\begin{tabular}{|c|c|c|c|c|c|c|c|c|c|c|c|}
\hline minspeed $(\mathrm{km} / \mathrm{h})$ & 0 & 5 & 10 & 15 & 20 & 25 & 30 & 35 & 40 & 45 & 50 \\
\hline Madrid (\% of ended simulations) & 56 & 100 & 100 & 100 & 100 & 100 & 100 & 100 & 100 & 100 & 100 \\
\hline Barcelona (\% of ended simulations) & 90 & 100 & 100 & 100 & 100 & 100 & 100 & 100 & 100 & 100 & 100 \\
\hline Zaragoza (\% of ended simulations) & 68 & 100 & 100 & 100 & 100 & 100 & 100 & 100 & 98 & 100 & 100 \\
\hline
\end{tabular}

\subsubsection{Monitoring process: results with $P H C A$}

If we test the same minimum speed threshold values with the variant PHCA of the mobile agent, we obtain another set of results, that are shown in Fig. 18. This version of the agent never uses spatial crowdsourcing when it is inside the target area, nor when the agent leaves it accidentally and must return to resume the monitoring task. The mobile agent only hops from car to car by using the MAP hop strategy to estimate which car in range will reach the target area earlier.

In Fig. 18(a), the total time is shown and the best results are, in general, for minimum speed threshold values around $5 \mathrm{~km} / \mathrm{h}$, whereas for higher values the total time increases very slightly.

The total number of hops is shown in Fig. 18(b), which exhibits a behavior similar to SCCA. For the extreme value of $0 \mathrm{~km} / \mathrm{h}$, the agent must only hop among the vehicles without using spatial crowdsourcing, and therefore the number of hops is much higher. For higher values of the minimum speed threshold, the number of hops decreases very slightly due to the use of spatial crowdsourcing to reach the target area and return to the origin.
The results of the amount of collected data, shown in Fig. 18(c), are noteworthy: all the results are only around $60 \%$, which is much lower than with SCCA. The reason is that, once the agent reaches the target area, it must visit the cells through only car-to-car hops, without using spatial crowdsourcing, which is harder to accomplish, as the number of vehicles inside the area is low and their trajectories unknown. Due to this, the agent needs more time to perform the task and in many cases it reaches the data collection timeout of 3 minutes even before it is completed.

In Fig. 18(d), the virtual money spent by the agent is shown. For the minimum speed threshold value of $0 \mathrm{~km} / \mathrm{h}$, the amount of money spent is 0 , since the agent never uses spatial crowdsourcing inside the target area, and neither to reach or leave it. For higher values, the best results are obtained for $5 \mathrm{~km} / \mathrm{h}$ and the worst results for values higher than $30 \mathrm{~km} / \mathrm{h}$.

Regarding the social cost shown in Fig. 18(e), we can observe the same behavior as with the SCCA version and again a very similar trend to the one shown in Fig. 18(d) for the virtual money 


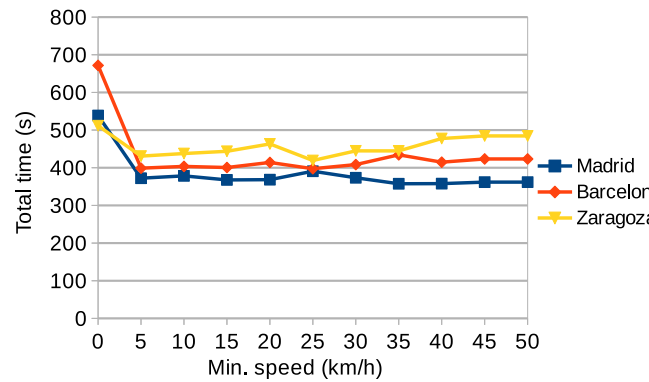

(a) Time

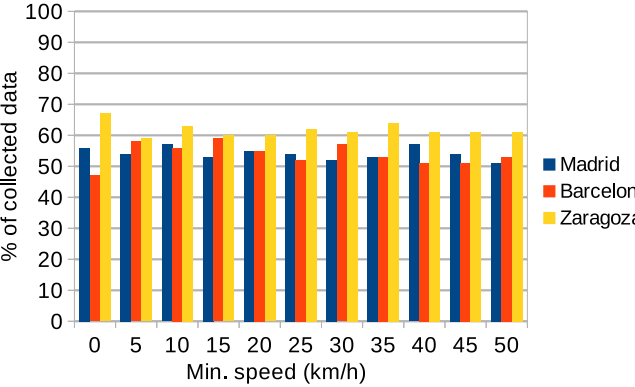

(c) Collected data

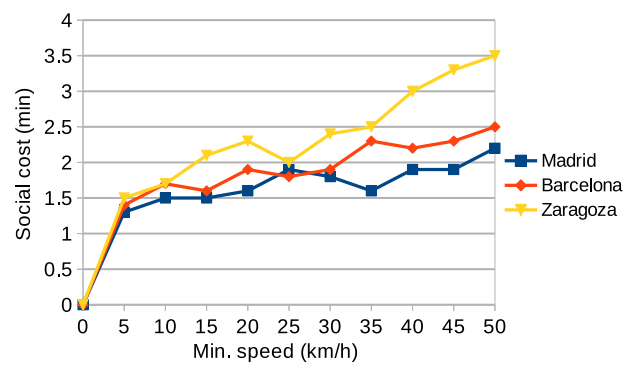

(e) Social cost

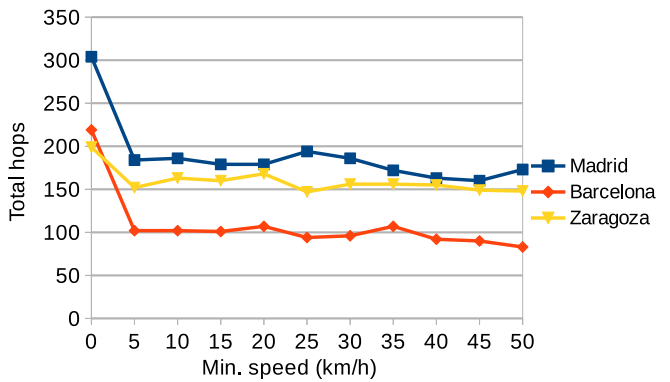

(b) Number of hops

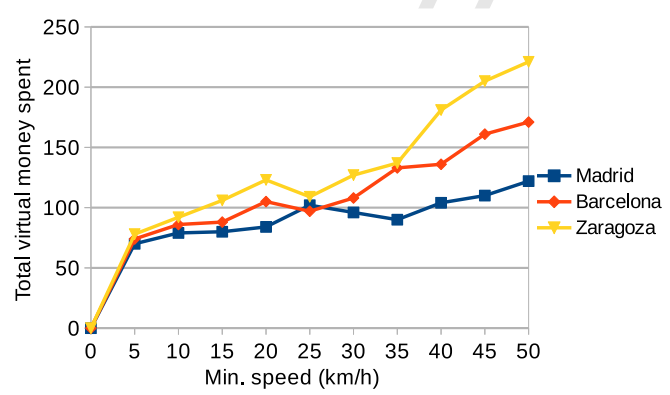

(d) Virtual money

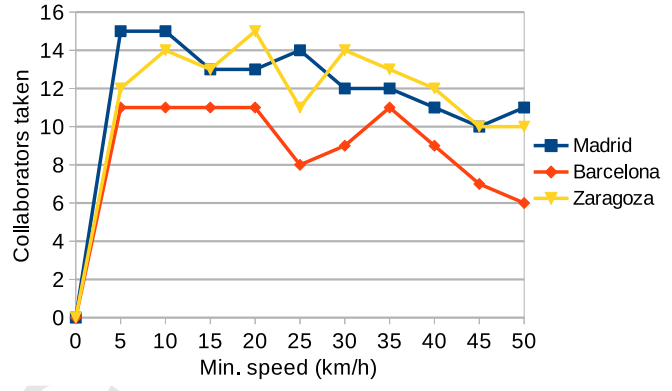

(f) Number of collaborators

Fig. 18. Monitoring process: results with PHCA.

Table 7

Monitoring process: reliability with PHCA.

\begin{tabular}{|c|c|c|c|c|c|c|c|c|c|c|c|}
\hline minspeed $(\mathrm{km} / \mathrm{h})$ & 0 & 5 & 10 & 15 & 20 & 25 & 30 & 35 & 40 & 45 & 50 \\
\hline Madrid (\% of ended simulations) & 68 & 98 & 100 & 100 & 100 & 100 & 100 & 100 & 100 & 100 & 100 \\
\hline Barcelona (\% of ended simulations) & 82 & 100 & 100 & 100 & 100 & 100 & 100 & 100 & 100 & 100 & 98 \\
\hline Zaragoza (\% of ended simulations) & 80 & 98 & 100 & 98 & 100 & 100 & 100 & 100 & 98 & 100 & 100 \\
\hline
\end{tabular}

spent by the agent, and thus the collaborators are compensated according to their actual cost in quite a fair way.

Finally, in Fig. 18(f), the number of collaborators taken by the agent is shown. This amount is exactly 0 for the minimum speed threshold value of $0 \mathrm{~km} / \mathrm{h}$, since spatial crowdsourcing is never used for traveling to/from the target area, nor for collecting data inside it when it is reached. For minimum speed threshold values of $5 \mathrm{~km} / \mathrm{h}$ and above, the collaborators are used in a similar way to the SCCA variant of the agent: as the minimum speed threshold grows, the number of collaborators decreases. Since the total time remains more or less constant, this means that for low values of the minimum speed threshold, a collaborator is usually taken for a smaller time than for higher threshold values.

Regarding the reliability of the whole process (shown in Table 7) the best results are for minimum speed threshold values between $10 \mathrm{~km} / \mathrm{h}$ and $45 \mathrm{~km} / \mathrm{h}$, with reliabilities of $100 \%$. On the other hand, the worst results are for the value of $0 \mathrm{~km} / \mathrm{h}$, when no spatial crowdsourcing is used, but there are also some cases when the reliability does not reach $100 \%$ and stays in $98 \%$.

\subsubsection{Monitoring process: comparison between SCCA and PHCA}

Comparing the results of these experiments (regarding the determination of the minimum approaching speed threshold), we can conclude that:

- The variant SCCA of the agent takes less time than PHCA to complete the monitoring process, due to the advantage of using spatial crowdsourcing inside the target area (on average, around $300 \mathrm{~s}$ in the first case and slightly more than $400 \mathrm{~s}$ in the second).

- Regarding the reliability, both approaches yield similar results, being not smaller than $98 \%$ for minimum approaching speeds of at least $5 \mathrm{~km} / \mathrm{h}$.

- Considering the total number of hops, SCCA is better (less than 100 hops, versus a value between 100 and 200 hops with PHCA) since the use of spatial crowdsourcing reduces the necessity to keep looking for potentially better vehicles to hop to. 
- Regarding the data collection rate, again SCCA outperforms PHCA, being the first one around $100 \%$ and the second one around $60 \%$.

- Concerning the virtual money cost, however, PHCA is better than the SCCA (with an average cost of 93 versus 126 units of virtual money), due to the fact that the former never uses spatial crowdsourcing once the mobile agent reaches the target area, which saves money.

- For both approaches, the use of spatial crowdsourcing with a minimum approaching speed threshold is always better than not using it, in terms of time, reliability, bandwidth usage and amount of collected data, as the results for the minimum speed threshold value of $0 \mathrm{~km} / \mathrm{h}$ show.

- For both variants, a minimum approaching speed threshold around $5 \mathrm{~km} / \mathrm{h}$ is appropriate, if we take into account all the results from these experiments.

Therefore, we can establish that the best algorithm in all terms (except in the virtual money cost) is SCCA, and we choose for the minimum approaching speed threshold $5 \mathrm{~km} / \mathrm{h}$, which is quite a low value. This value, as well as the SCCA strategy, are used by default in the experiments, as shown in Table 3.

\subsection{Experiments analyzing the impact of different factors on the monitoring process}

In the third set of experiments, we analyze the impact of different parameters on the performance of the whole monitoring process, such as the percentage of collaborators (Section 4.4.1), the existing traffic density (Section 4.4.2), and the required minimum stay time of mobile agents in the vehicles (Section 4.4.3). In these experiments, the mobile agent follows the complete monitoring process described in Section 3: it first travels to the interest area and, once the interest area is reached, it visits every cell into which it is divided to read the required data by using the available sensors in its carrying vehicle. When the mobile agent has read all the sensors required (or the maximum timeout expires), it returns to its origin carrying with it the data collected. As in Section 4.3, the probability that the trajectory of a given vehicle will travel inside the interest area is $10 \%$, as in this case the use of spatial crowdsourcing for the data collection stage (SCCA approach) can be more relevant.

\subsubsection{Impact of the percentage of potential collaborating vehicles}

Fig. 19(a) shows how the total time needed to complete the whole monitoring process varies with the ratio of vehicles willing to act as collaborators in spatial crowdsourcing tasks. The required time is around 500 to 700 seconds for $0 \%$ of potential collaborators, that is, when no spatial crowdsourcing can be used and the mobile agent can only rely on hopping from one vehicle to another to reach the destination and perform the monitoring task inside the target area. The time decreases to around 400 seconds when the ratio of potential collaborators increases to $10 \%$, and then decreases slightly until it reaches around between 300 and 350 seconds for $100 \%$ collaborators (i.e., all the vehicles in the scenario can act as collaborators). The reason is that once the mobile agent finds a suitable collaborator it is not necessary to keep looking for another one, and thus having more potential collaborators to choose from is useless.

Figs. 19(b), 19(c) and 19(e) show the number of hops performed by the mobile agent, the amount of data collected, and the social cost, respectively. In all these figures, we can see the same pattern: when spatial crowdsourcing is not used (because the ratio of potential collaborators is $0 \%$, then the corresponding metric measured is significantly worse than when it is used.
The results improve dramatically with only about $10 \%$ collaborators. However, when the ratio of potential collaborators is higher than this value, the measured parameters do not show further improvement (the mobile agent does not significantly benefit from a higher number of potential collaborators). The exception to this pattern is the virtual money spent, shown in Fig. 19(d), which is obviously 0 when no collaborators are used for performing spatial crowdsourcing. Similarly to what was observed in previous experiments, as expected, Figs. 19(e) and 19(d) show a similar pattern.

Regarding the number of collaborators used by the agent, shown in Fig. 19(f), it is obviously 0 when the ratio of potential collaborators is $0 \%$. For higher ratios, the number of effective collaborators used by the agent varies more or less randomly, but for all the cases its number is within the range of 10 to 21 collaborators. When the mobile agent commutes from spatial crowdsourcing to hopping to other vehicles and back again to spatial crowdsourcing, it seems to find a new collaborator easily, as the total time for the monitoring (shown in Fig. 19(a)) does not increase significantly.

In Table 8 , we can see how the reliability is between $68 \%$ and $80 \%$ (depending on the city map) when the ratio of potential collaborators is $0 \%$. For $10 \%$ of potential collaborators, it increases to between $96 \%$ and $100 \%$, and for higher values the reliability is always $100 \%$, with the exception of a few cases where it is $98 \%$. Therefore, not being able to use spatial crowdsourcing (i.e., $0 \%$ of potential collaborators) makes the process more unreliable than when using it.

As a conclusion for this experiment, we can say that the benefits of using spatial crowdsourcing are remarkable even with a ratio of potential collaborators as low as $10 \%$. Therefore, we do not need a high number of drivers willing to modify their trajectories to benefit from the spatial crowdsourcing approach and increase the performance of the process.

\subsubsection{Impact of the density of vehicles}

In this experiment, we test the influence of the density of vehicles on the performance of the monitoring process. In the previous experiments, where the density was set to 100 vehicles $/ \mathrm{km}^{2}$ (a medium value), the results showed that even with a small number of potential collaborators the performance improves dramatically. However, we also want to test it in a more challenging scenario with low and very low vehicle density, to find out the limits of spatial crowdsourcing. The chosen density values and their denomination (low density, medium density, etc.) are inspired by those used in proposals such as [37,38], and for this experiment we vary them from 10 vehicles $/ \mathrm{km}^{2}$ (very low density) to 100 vehicles $/ \mathrm{km}^{2}$ (medium density). Based on the results of the experiment presented in Section 4.4.1, in this experiment the percentage of potential collaborating vehicles is set to $10 \%$.

In Fig. 20(a), the total time to complete the monitoring process is shown. As the number of vehicles increases, the time needed decreases, with little variance when the density reaches around between 70 and 80 vehicles $/ \mathrm{km}^{2}$. The reason is that, with a high number of vehicles, the mobile agent has less difficulties to find a car to be carried nearer the target area, whether it uses spatial crowdsourcing or just hops from one vehicle to another.

Regarding the number of hops performed by the mobile agent, shown in Fig. 20(b), its number increases with the vehicle density, since the agent is constantly looking for a better option to reach its destination sooner: if there exist more vehicles, then the number of opportunities is also higher and the agent will likely hop to another car more frequently.

The amount of data collected within the timeout of 3 minutes, shown in Fig. 20(c), increases with the vehicle density, although there is little variation and all the values are higher than $90 \%$. 


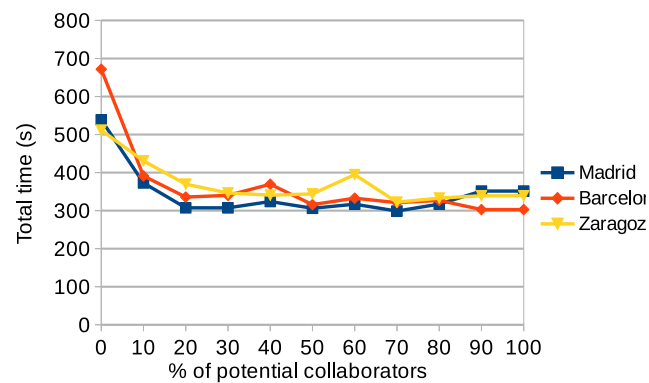

(a) Time

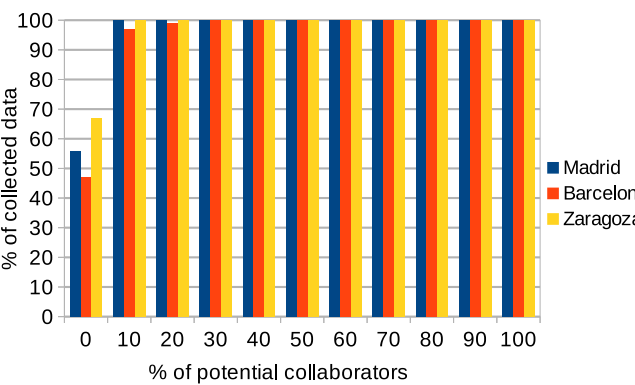

(c) Collected data

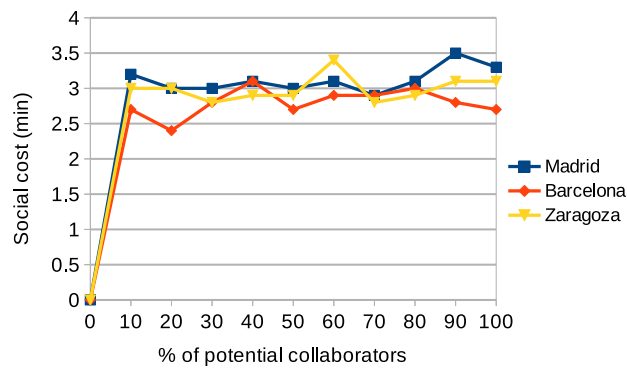

(e) Social cost

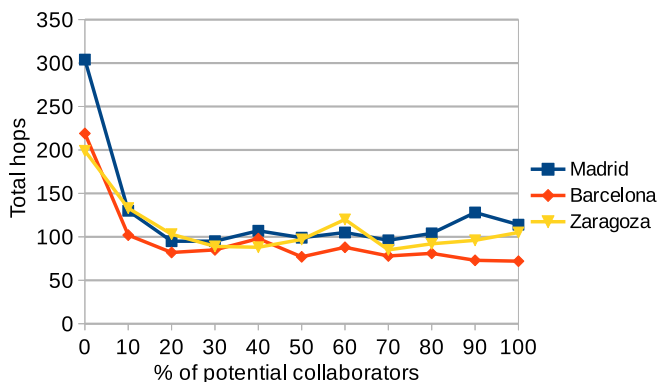

(b) Number of hops

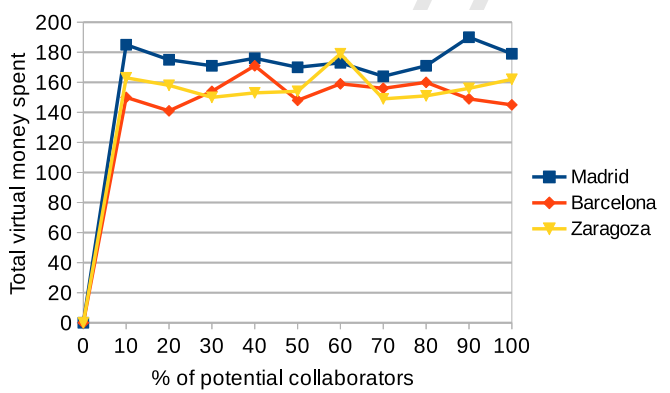

(d) Virtual money

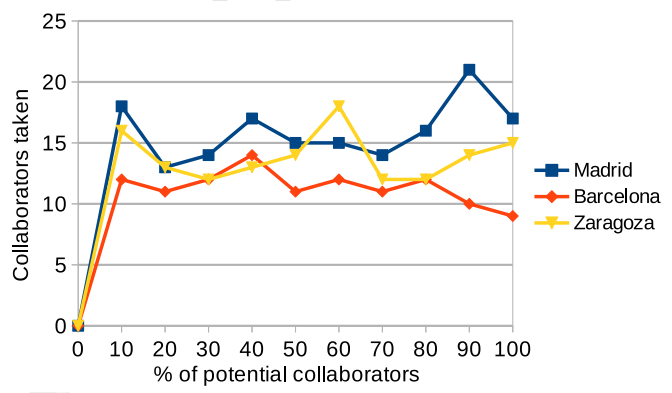

(f) Number of potential collaborators

Fig. 19. Monitoring process: results varying the percentage of potential collaborating vehicles.

Table 8

Monitoring process: reliability varying the percentage of collaborating vehicles.

\begin{tabular}{|c|c|c|c|c|c|c|c|c|c|c|c|}
\hline$\%$ of collaborators & 0 & 10 & 20 & 30 & 40 & 50 & 60 & 70 & 80 & 90 & 100 \\
\hline Madrid (\% of ended simulations) & 68 & 100 & 100 & 100 & 98 & 100 & 100 & 98 & 100 & 98 & 100 \\
\hline Zaragoza (\% of ended simulations) & 80 & 96 & 100 & 98 & 100 & 100 & 100 & 98 & 100 & 100 & 100 \\
\hline
\end{tabular}

Figs. 20(d) and 20(e) show the virtual money paid by the mobile agent to the collaborators and the social cost, respectively. As in the previous experiments, these figures are closely related, showing the relation between the social cost and the virtual money spent by the mobile agent, and both values increase slightly with the density of vehicles. With a higher number of vehicles there is also a higher number of potential collaborators, and the agent can obtain help more easily, and therefore it could end up spending more virtual money. On the other hand, with less vehicles there is a smaller number of potential collaborators, so it is more likely that the agent cannot find one of them when needed and, consequently, it might spend less virtual money.

Finally, the number of collaborators taken by the mobile agent, shown in Fig. 20(f), increases significantly with the density of vehicles, for the same reasons: since the number of potential collaborators is higher, the agent has more opportunities to take advantage of them and they are used more frequently.

Table 9 shows the reliability of the monitoring process for the different traffic density values. As the vehicle density increases, so does the reliability, and starting with 50 vehicles $/ \mathrm{km}^{2}$ there is little variance. This result is related to the total time required for the monitoring, as the sooner the mobile agent completes all the phases of the process, the less likely the time limit will be reached.

As a conclusion for this experiment, we can say that, regarding the influence of the vehicle density in spatial crowdsourcing, the best results in terms of time, reliability and amount of collected data are obtained for values around 60 vehicles $/ \mathrm{km}^{2}$ (a low density) and higher. For lower densities, the results are worse, but not so bad for the spatial crowdsourcing to be considered useless under those circumstances: for example, for the extremely low density value of 10 vehicles $/ \mathrm{km}^{2}$ the amount of collected data is higher than $90 \%$ and the reliability is near $75 \%$ in the worst case, which may be enough for monitoring tasks with soft requirements.

\subsubsection{Impact of the minimum stay time in a collaborator}

In this experiment, we evaluate the impact of the minimum stay time required in each collaborating vehicle used. Specifically, we vary this value from 0 to 50 seconds in increments of 


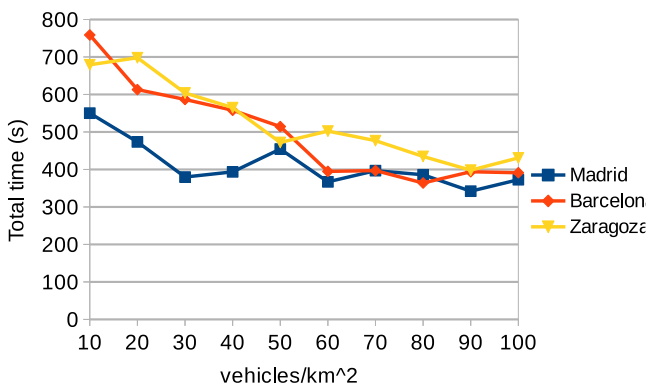

(a) Time

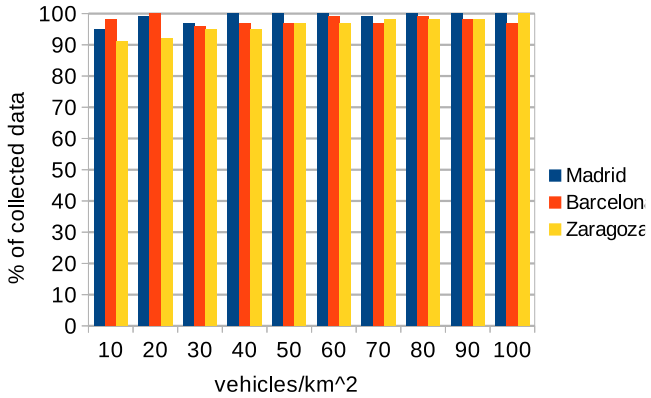

(c) Collected data

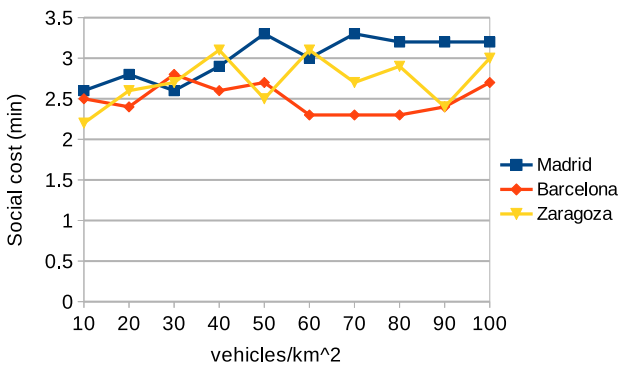

(e) Social cost

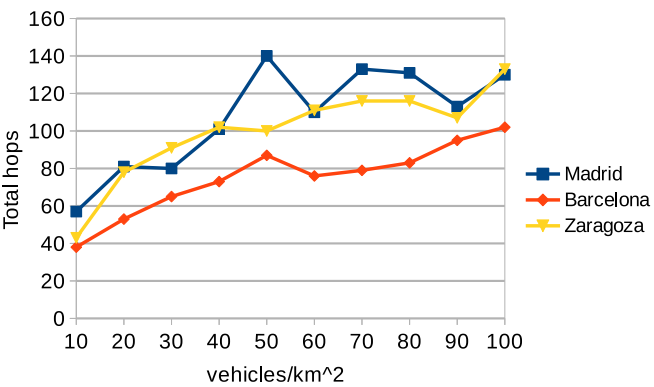

(b) Number of hops

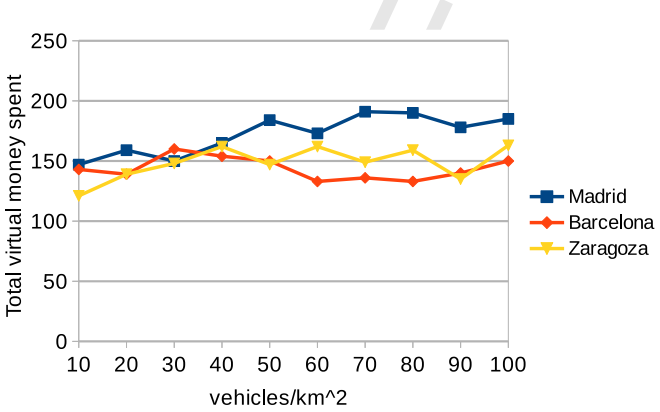

(d) Virtual money

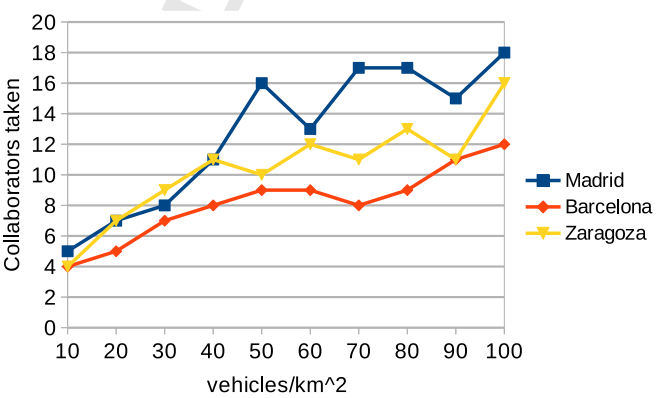

(f) Number of collaborators

Fig. 20. Monitoring process: results varying the density of vehicles.

Table 9

Monitoring process: reliability varying the density of vehicles.

\begin{tabular}{|c|c|c|c|c|c|c|c|c|c|c|}
\hline Density (vehicles $/ \mathrm{km}^{2}$ ) & 10 & 20 & 30 & 40 & 50 & 60 & 70 & 80 & 90 & 100 \\
\hline Madrid (\% of ended simulations) & 86 & 98 & 98 & 100 & 98 & 100 & 98 & 100 & 98 & 100 \\
\hline Barcelona (\% of ended simulations) & 74 & 86 & 100 & 92 & 96 & 98 & 100 & 100 & 100 & 100 \\
\hline Zaragoza (\% of ended simulations) & 80 & 82 & 96 & 88 & 98 & 100 & 100 & 100 & 100 & 96 \\
\hline
\end{tabular}

5 seconds, to evaluate its impact. Besides, based on the results of the experiment presented in Section 4.4.1, in this experiment the percentage of potential collaborating vehicles is set to $10 \%$. In Fig. 21(a), we can see how the total time to complete the process decreases slightly as the minimum stay time increases. The reason is that the likelihood of wasting time decreases when the agent stays in a collaborator longer, as the collaborator commits itself to bring the agent to its destination. Besides, the number of hops also decreases, as shown in Fig. 21(b).

Regarding the amount of collected data, shown in Fig. 21(c), it remains more or less constant as the minimum stay time increases, and it is always above $96 \%$. The mobile agent takes into account the minimum stay time only in the phases of the process where it approaches the target area and returns to its origin, but not in the data gathering phase, so it has no influence during the data collection step.

The invested virtual money and the social cost, shown in Figs. 21(d) and 21(e), respectively, exhibit a similar behavior, like in the previous experiments, in this case both growing with the minimum stay time. The difference between the values observed for the lowest minimum stay value (0 seconds) and the highest minimum stay value (50 seconds) is about $25 \%$, and the reason is that the mobile agent wastes less time in hops to other vehicles and stays longer in the collaborating vehicles, so these collaborators receive more compensations in the form of virtual money. This fact is confirmed by the number of collaborating vehicles taken by the agent, shown in Fig. 21(f). As the minimum stay time grows, the number of collaborators decreases strongly. Along with the low variability of the total time, this means that the mobile agent takes a smaller number of collaborators but stays longer in them.

As shown in Table 10 , the reliability parameter is $100 \%$ for almost all the minimum stay times (with only one exception, with $96 \%$, for the city of Zaragoza and a minimum stay time of 0 seconds). The reliability is related to the total time of the process (and whether it takes longer than the timeout or not), and given the 


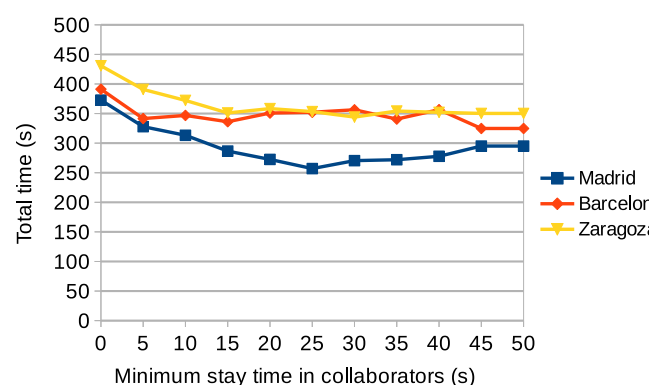

(a) Time

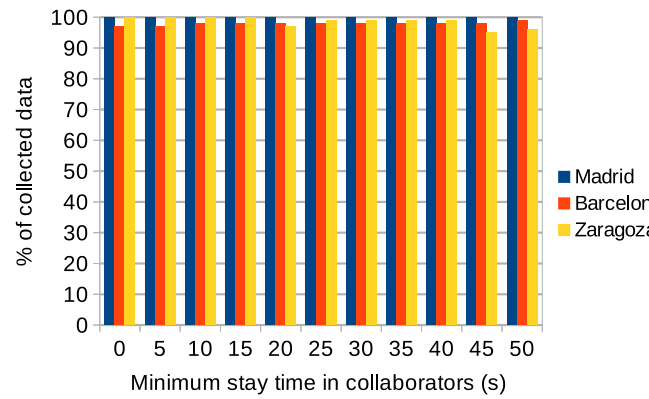

(c) Collected data

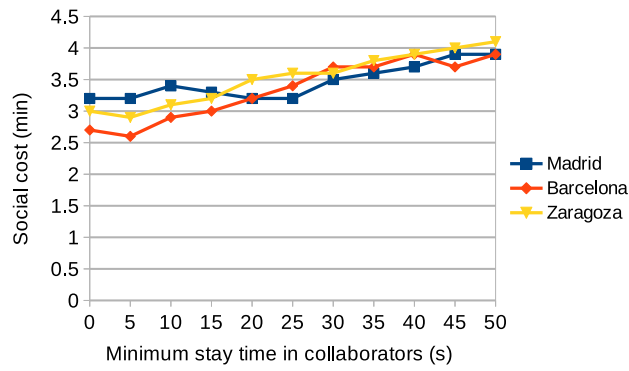

(e) Social cost

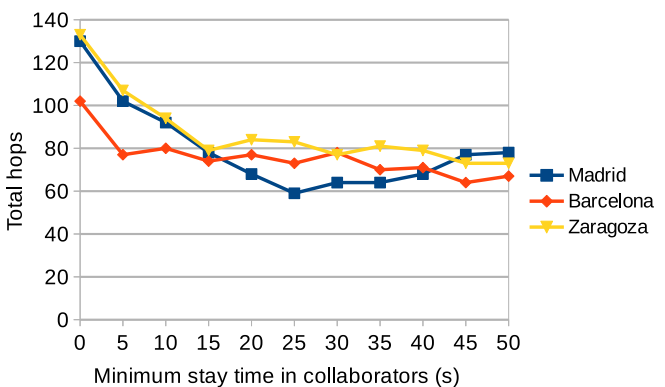

(b) Number of hops

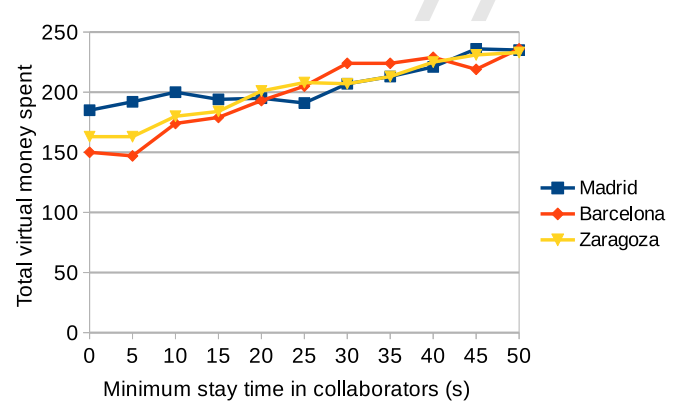

(d) Virtual money

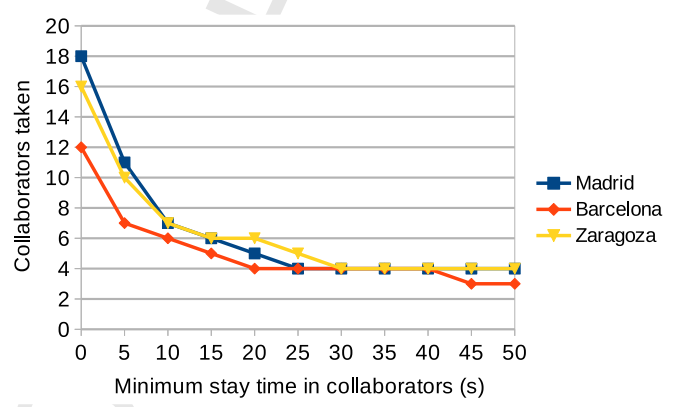

(f) Number of collaborators

Fig. 21. Monitoring process: results varying the minimum stay time of the mobile agent in collaborators.

Table 10

Reliability varying the minimum stay time of the mobile agent in collaborators.

\begin{tabular}{|c|c|c|c|c|c|c|c|c|c|c|c|}
\hline Minimum stay time (s) & 0 & 5 & 10 & 15 & 20 & 25 & 30 & 35 & 40 & 45 & 50 \\
\hline Madrid (\% of ended simulations) & 100 & 100 & 100 & 100 & 100 & 100 & 100 & 100 & 100 & 100 & 100 \\
\hline Barcelona (\% of ended simulations) & 100 & 100 & 100 & 100 & 100 & 100 & 100 & 100 & 100 & 100 & 100 \\
\hline Zaragoza (\% of ended simulations) & 96 & 100 & 100 & 100 & 100 & 100 & 100 & 100 & 100 & 100 & 100 \\
\hline
\end{tabular}

small variance of the total time, the minimum stay time has no appreciable effect on the reliability.

As a conclusion, the use of a minimum stay time in a collaborator has a certain influence on the number of hops that the agent needs to take, decreasing them as the minimum stay time grows. However, this reduction has no influence on the reliability or the amount of collected data, and increases the social cost of the collaborators and the amount of virtual money spent by the agent.

\section{Related work}

Spatial crowdsourcing is a hot topic nowadays. There exist some proposals related to monitoring and sensing in urban areas by using vehicles and networks but, up to the authors' knowledge, our work is the first proposal that applies the idea of spatial crowdsourcing to vehicular networks, with the exception of our previous seminal work that was published as a short paper [9]. Moreover, as opposed to other works focusing on spatial crowdsourcing in general, our proposal is completely distributed and opportunistic, as it does not rely on a central entity with global knowledge about the potential participants and benefits from the direct interactions among nearby vehicles. By opportunistically considering neighbor vehicles as potential collaborators, the disadvantages of centralized approaches are avoided. In this section, we compare some related work and highlight the major differences and enhancements of our proposal. We first consider approaches related to spatial crowdsourcing in general and then approaches in the specific context of vehicles.

\subsection{Related work on spatial crowdsourcing and crowdsensing}

The topic of mobile crowdsensing (also called collaborative sensing, mobile sensing, or participatory sensing) has attracted significant research attention (e.g., see [39-45]).

One common problem of spatial crowdsourcing is known as the Maximum Task Assignment (MTA) problem, which focuses on how to allocate the available workers (in our context, collaborating vehicles) to spatial tasks, to maximize the global performance. There 
exist many proposals to tackle this problem (e.g., see [46-51]) under different circumstances and with different constraints. However, most of them need a central server which centralizes all the tasks that need to be assigned and which constantly receives the updated location of the potential collaborators, in order to allocate them the most appropriate task according to several factors (e.g., their position, travel time to the destination, etc.). These solutions have the advantage of optimizing the performance of the crowdsourcing process, but they also have some drawbacks, such as privacy concerns (the central server needs to know the position of all the collaborators) and the need of direct communications with the central server, which can have an economic cost (in case of using 3G/4G technologies) or may need a previously-existing infrastructure (e.g., cellular phone towers or Wi-Fi access points). In our approach, we consider a pure ad hoc solution where a mobile agent directly asks for help to the neighbor vehicles. Thus, we avoid the disadvantages of the centralized approaches. However, the assignments performed in our approach are not necessarily globally optimal, as they are based on local decisions taken without having a global vision of the scenario.

In [52], the authors propose a method for recruiting collaborators for crowdsensing tasks, by using semi-Markov models to predict the trajectory that the potential collaborators will follow and to estimate if recruiting them may be interesting or not according to the existing sensing needs. In our approach, there is no explicit recruiting process based on the trajectories of the vehicles, which we assume that can be unknown (e.g., due to privacy reasons): only vehicles in the neighborhood (within the communication range) of the vehicle carrying the mobile agent can participate in the spatial crowdsourcing, and they may accept or not a potential collaboration when the agent asks for their help. Nevertheless, the proposal in [52] could be used as a complementary technique if access to those trajectories is enabled.

In [41], the authors present a sensing engine called MoST (Mobile Sensing Technology), that can be used as a framework for developing location-aware applications for Android mobile phones. The data read by the mobile phone sensors are transferred to a central server by using the connection available on the phone (Wi-Fi, $3 \mathrm{G} / 4 \mathrm{G}$, etc.) and processed in the server. This work is not related specifically to VANETs, but it illustrates the interest of sensing and crowdsourcing applications.

In [53], the authors tackle the problem of recruiting vehicles for public sensing in such a way that the selected vehicles cover an area as large as possible at the lowest cost. To achieve this, they present a reputation-aware, trajectory-based recruitment (RTR) framework that handles the recruitment of vehicles for public sensing. The framework considers the spatiotemporal availability of participants along with their reputation to select vehicles that should achieve the desired coverage of an area of interest with a cost within the budget limitations. The framework consists of a reputation assessment scheme, a pricing model, and a selection scheme, that are combined to accomplish the objective of maximizing the coverage with the minimum cost. The authors propose greedy heuristic solutions targeting the selection problem in realtime. The RTR framework generalizes the basic selection problem to handle some practical scenarios, including vehicles that leave the area that needs to be monitored, and varying redundancy requirements. An extensive performance evaluation shows that the proposed greedy heuristics are able to achieve results close to those previously obtained by optimal benchmarks under different scenarios, and that the framework succeeds in achieving high levels of coverage even when the vehicles do not stick to their announced trajectories. Another work related to the recruitment of collaborators is [54], where the authors propose a model based on genetic algorithms that selects the most appropriate group of participants using parameters related to the users, the devices, and the interest area. In our spatial crowdsourcing approach, a mobile agent takes decisions locally based on information regarding the neighbor vehicles, and therefore the collaboration takes place opportunistically rather than through an explicit recruitment process.

It might also be possible to consider more sophisticated crowdsourcing strategies with the goal to minimize the social cost while at the same time ensuring a minimum compensation for the driver. For example, in the context of ride-sharing for passengers, [55] provides a method to select a driver to maximize the overall shared route percentage (SRP) subject to a minimum required value of this parameter for each driver (expectation rate of the driver). This technique could be applied to locate vehicles that, even if they are not at that moment within communication range, could pick up the mobile agent and carry it to the intended destination. However, applying this method in a vehicular ad hoc network, where there is no global view of the environment and the vehicles are usually constantly moving, is challenging.

Other works related to crowdsensing, but further from the specifics of the spatial crowdsourcing approach described in this paper, are [56,57]. In [56], the authors propose GENIUS-C, a framework to support the development of spatial crowdsourcing platforms. It is based on a generic architecture to reduce the gap between academy and industry, and is meant to decrease the development cost and effort and increase the overall quality of spatial crowdsourcing platforms. A case study is created using GENIUS-C to demonstrate its benefits and how it can be used in the development of spatial crowdsourcing platforms. In [57], the authors describe an algorithm to detect the accuracy of data that come from crowdsensing sources such as sensors aboard vehicles (car sensors), sensors built into mobile devices (phone sensors) carried by one or more occupants, or both. They demonstrate, through evaluation in real environments (using infrastructure that captures car and phone sensor readings and uploads them to a cloud database), that their detection algorithms can achieve high accuracy for some tasks related to the driver's behavior and the environment (e.g., higher than $90 \%$ for lane change determination) and that crowdsensing plays an indispensable role in improving the detection performance (e.g., improving recall by $35 \%$ for lane change determination on curves). These are complementary works to ours, as they focus on different issues related to crowdsensing.

Besides, although it does not specifically propose a crowdsensing method, another relevant related work to mention is [58], where the authors describe a hierarchical Bayesian non-parametric approach for efficient and scalable route prediction, that can harness the wisdom of crowds of route planning agents by aggregating their sequential routes of possibly-varying lengths and origindestination pairs. This approach has the advantages that it does not require a Markov assumption and that it generalizes well with sparse data, thus resulting in an improved prediction accuracy, as the authors demonstrate empirically using real-world data about trajectories of taxis.

Finally, regarding the use of incentives to encourage cooperation, OPPay [59] is a payment system for opportunistic data services (such as Wi-Fi sharing, content-based file sharing, and opportunistic networking), which implements a micropayment communication protocol for mobile devices to perform data transactions and make payments using bitcoins. OPPay is intended to operate using incremental payments that are resilient to interruptions in the communications, and therefore it may be of interest in the context of vehicular networks. Another interesting work regarding this topic is presented in [60], where the authors propose a rewarding mechanism for mobile crowdsourcing applications based on auctions and biddings of the collected data, that focuses on preserving the privacy of the collaborators in order to encourage their participation. In our approach, we propose the use of virtual money to pay collaborating vehicles, but the specific details 
about how those payments can be made is out of the scope of this paper. Other economic and incentive models have been proposed for mobile P2P networks (e.g., [61,62]) and collaborative sensing in general (e.g., [63-66]).

\subsection{Related work on monitoring tasks with vehicles}

The monitoring of certain parameters (e.g., polluting gases and particles, pollen, or solar radiation) has been performed traditionally using fixed sensing stations, that have a number of disadvantages, such as their lack of flexibility to perform a monitoring task different from the one considered initially (e.g., monitor a different area), and the requirement of a certain infrastructure to provide the energy and communication support required, which also make them costly to build and maintain. As an alternative, vehicles can be used as mobile monitoring platforms, since they can be equipped with the necessary sensors and can travel to any place (as long as a nearby road exists) to monitor the required parameters. The sensors installed can be replaced by others if it is required to monitor different parameters. Besides, the sensors do not need a dedicated power or communication infrastructure, since they can use the power generated by the vehicle's engine and the wireless communication devices of the on-board computer. For these reasons, the use of vehicles for performing monitoring tasks has attracted the attention of the research community, and the term vehicular sensor network has emerged $[67,68]$. In the following, we present some representative works related to this topic, in order to illustrate a variety of monitoring tasks that have attracted research attention.

In [69], the authors assume the existence of a vehicular network in which the vehicles carry sensors, and they pose the problem of determining how much information should be received from the sensors and where should they be located to achieve an optimum monitoring process. If the sensors send information very frequently, then their readings would likely be similar, which implies that bandwidth would be wasted. On the other hand, if the sensors send a little amount of information, then the measurements may be not accurate enough. That work is centered on the mathematical aspect of such a method. It is considered that sensors send their data to a central server by using cellular connections instead of ad hoc communications. As stated before, and also in studies such as [4], this has some disadvantages, such as the existence of economic cost for the user.

In [70], the authors use information about the traffic status to compute the best route to recharging points for electrical vehicles, taking also into account the amount of energy available on board. The traffic status is received from the surrounding cars, that send information about their position and speed to estimate if the traffic is light or dense. Ad hoc communications and a simple flooding model for data dissemination is used. However, the proposal focuses only on sharing traffic data. In our proposal, instead, the data sent by the vehicles can be of any type (not only the speed and position of vehicles) and the sensed data are carried out by mobile agents, which can behave more efficiently than flooding algorithms that continuously send all the data to all the neighbors. So, a mobile agent can encapsulate any desired dissemination approach and even commute from one approach to another depending on context conditions (e.g., the density of vehicles in the area). With the approach presented in this paper, only a limited amount of data is sent, every time a mobile agent hops from one vehicle to another, instead of sending multiple copies of the data to multiple receivers, as it occurs with flooding algorithms. Besides, the proposal in [70] does not exploit any spatial crowdsourcing approach.

A working prototype is presented in [71] that consists of a monitoring VANET where the vehicles are equipped with sensors and wireless communication devices that constantly look for Wi-
Fi access points to opportunistically send the collected data to a central server. By contrast, in our approach the data are stored in the vehicles instead of being all sent to a central server, and they are processed distributively by mobile agents searching for the most relevant data to perform the monitoring task. The system presented in [71] was deployed in a real city and the vehicles participating in the VANET belonged to the local authority, instead of being open to any citizen, which has both advantages and drawbacks.

In [72], the authors propose a method for route planning in a city by using information collected opportunistically by the moving vehicles. The system operates in a decentralized way and it exploits a technique based on the ant optimization problem, which leads to routes that are not strictly optimal (as opposed to classic shortest path search algorithms) but good enough to both reach the intended destination and distribute the traffic flows along different streets so that they do not become congested. While the approach is interesting, its application is limited to the problem of route planning and its performance in low-traffic density scenarios is not clear.

In [73], a smart parking system for locating available parking slots is proposed. In this system, end users are not the only subjects that collaborate by sharing information. Additionally, both the parking controllers and the city administrators also participate by providing information in a fast and integrated way. However, the system operates in a centralized way, and a mobile 3G/4G connection is required to operate it.

In [74], a probabilistic vehicle routing algorithm is proposed, based on a variant of the $A^{*}$ algorithm, to improve the sensing coverage of vehicles. The idea is to reduce the optimality of $A^{*}$ regarding the computation of shortest paths in exchange for a better sensing coverage. As opposed to our proposal, special vehicles (called probe vehicles) are considered to perform the spatial crowdsourcing (in particular, taxis) rather than conventional cars. Besides, the approach presented in [74] is not based on mobile agents and has to be predefined in advance. Finally, it requires a centralized entity to compute the paths, as global knowledge of the sensing area covered and the trajectories is required.

In [75], the authors present a unified delay analysis framework for opportunistic data collection, that integrates the sensing and transmission delays, that are usually analyzed separately. A third delay metric (that they call data collection delay) is added to the analysis that can be performed by the framework, and thus the QoS of opportunistic data collection applications can be measured more comprehensively. The theoretical analysis of the framework is validated by the authors by performing a number of simulations. This work is complementary to ours, as it focuses on the analysis of performance metrics.

Also related to delay aspects, in [76], the authors tackle the problem of the sensor's reading delay, and they propose a solution to mitigate it. Certain types of sensors (for example, some gas sensors) take a relatively long time (about 20 to 50 seconds) to reach a stable state and obtain a valid sample measurement. If these sensors are carried by moving vehicles, they may have moved several hundred meters in that lapse, thus obtaining data from a place which is distant from the intended target. If this effect is not taken into account, the measured parameters may be treated incorrectly and they could lead to wrong conclusions. To avoid this, the authors propose a method to calibrate the sensor readings by using a filter on the raw data provided by the sensors, which also has as a benefit the reduction of the time needed to obtain valid sensors readings.

Finally, a recent work [77] analyzes crowdsourcing in Intelligent Transportation Systems (ITS), but it does not focus on vehicular networks nor spatial crowdsourcing. As another example, [78] proposes a privacy-preserving vehicular urban sensing platform; how- 
Table 11

Summary of advantages and disadvantages of several spatial crowdsourcing (SC) strategies.

\begin{tabular}{|c|c|c|c|}
\hline SC strategy & Description & Advantages & Disadvantages \\
\hline SC/PHCA & Pure hopper collecting agent & It needs less collaborators & $\begin{array}{l}\text { The collecting data task in low-density traffic areas can } \\
\text { be challenging }\end{array}$ \\
\hline SC/SCCA & Spatial crowdsourcing collecting agent & It can collect more data in low-density traffic areas & It needs more collaborators \\
\hline Without SC & It does not use SC & It does not need to compensate drivers & $\begin{array}{l}\text { The whole monitoring process in low-density traffic areas } \\
\text { can be challenging }\end{array}$ \\
\hline
\end{tabular}

ever, it relies on a cellular network, rather than when using ad hoc communications, and focuses on privacy issues and not on the use of spatial crowdsourcing.

\section{Conclusions and future work}

In this paper, we have presented a method that uses mobile agents for monitoring data in vehicular networks using spatial crowdsourcing. Specifically, in order to enhance the efficiency of the mobile agents when they perform their tasks, an agent has the possibility of requesting other collaborating users to modify their vehicles' routes to transport the agent to a more convenient place (i.e., closer to the target area) when its approaching speed is too low. We have performed an extensive set of tests by using a realistic simulator of vehicular networks and real road maps, and the results obtained show that the use of the proposed spatial crowdsourcing technique can increase the performance of the monitoring process, allowing the agent to reach the interest area faster, with a more efficient use of the wireless communications bandwidth and a higher data collection rate than when spatial crowdsourcing is not used. We quantify the results and precisely determine the extent of the improvement, its costs, and under which conditions those benefits are obtained. According to the experimental results presented, the proposed strategy is suitable even in conditions with a good traffic of vehicles, as it adapts itself to the existing conditions in order to make an efficient use of collaborators, commuting between using collaborators and hopping as needed. Therefore, using the spatial crowdsourcing approach presented is highly beneficial in low-traffic scenarios and it does not affect adversely the time performance of the monitoring process in any situation. Nevertheless, the experimental results show that several parameters could be adjusted depending on the existing constraints and preferred metrics. For example, if in the application scenario the agent is needed to spend as little virtual money as possible, then the minimum approaching speed threshold and the minimum stay time should be set to low values, although the amount of data collected and the reliability of the process will likely be negatively affected. Table 11 shows a summary with the main advantages and disadvantages of the different spatial crowdsourcing strategies considered.

As future work, we would like to analyze the use of possible variants and extensions of our proposed spatial crowdsourcing techniques and monitoring strategies and evaluate them in a wide variety of scenarios. Besides, it is interesting to study in more detail different payment methods that could be considered and their impact, considering desired qualities regarding the reliability, security, privacy, fairness and trustfulness of the system. The promising results that we have obtained with this approach encourages us to continue this research line and explore these and other possibilities.

\section{Acknowledgements}

This research has been supported by the projects TIN2016-78011-C4-3-R (AEI/FEDER, UE) and DGA-FSE (COS2MOS research group). We also thank the support of the TRAFAIR project
(2017-EU-IA-0167), co-financed by the Connecting Europe Facility of the European Union. The contents of this publication are the sole responsibility of its authors and do not necessarily reflect the opinion of the European Union. The maps of the cities used in the experimental evaluation are derived from data obtained from OpenStreetMap. Last but not least, we thank the reviewers for their useful and insightful comments, that have helped to improve this paper.

\section{References}

[1] J.J. Blum, A. Eskandarian, L.J. Hoffman, Challenges of intervehicle ad hoc networks, IEEE Trans. Intell. Transp. Syst. 5 (4) (2004) 347-351.

[2] H. Hartenstein, L.P. Laberteaux, A tutorial survey on vehicular ad hoc networks, IEEE Commun. Mag. 46 (6) (2008) 164-171.

[3] S. Olariu, M.C. Weigle, Vehicular Networks: From Theory to Practice, 1st edition, Chapman \& Hall/CRC, 2009.

[4] S. Ilarri, T. Delot, R. Trillo-Lado, A data management perspective on vehicular networks, IEEE Commun. Surv. Tutor. 17 (4) (2015) 2420-2460.

[5] B. Fleming, Sensors - a forecast [automotive electronics], IEEE Veh. Technol. Mag. 8 (3) (2013) 4-12.

[6] ABI Research, Sensor data crowdsourcing to transform connected car services, https://www.abiresearch.com/press/sensor-data-crowdsourcing-transformconnected-car-services/, May 2018, last access on February 6, 2019.

[7] ABI Research, Transformative connected car services built on data crowdsourcing, https://www.abiresearch.com/market-research/product/1027945transformative-connected-car-services-buil/, 2019, last access on February 6.

[8] O. Urra, S. Ilarri, R. Trillo-Lado, An approach driven by mobile agents for data management in vehicular networks, Inf. Sci. 381 (2017) 55-77.

[9] O. Urra, S. Ilarri, Towards spatial crowdsourcing in vehicular networks using mobile agents, in: 20th East-European Conference on Advances in Databases and Information Systems (ADBIS), vol. 637, Springer, 2016, pp. 88-95.

[10] L.-W. Chen, Y.-C. Tseng, K.-Z. Syue, Surveillance on-the-road: vehicular tracking and reporting by V2V communications, Comput. Netw. 67 (2014) 154-163.

[11] J. Zuo, H. Xia, S. Liu, Y. Qiao, Mapping urban environmental noise using smartphones, Sensors 16 (10) (2016) 1692.

[12] S. Mathur, T. Jin, N. Kasturirangan, J. Chandrasekaran, W. Xue, M. Gruteser, W. Trappe, ParkNet: drive-by sensing of road-side parking statistics, in: Eighth International Conference on Mobile Systems, Applications, and Services, MobiSys, ACM, 2010, pp. 123-136.

[13] Inria, SoundCity: a mobile application for understanding your exposure to noise pollution, https://mimove.inria.fr/soundcity, 2019, last access on February 6.

[14] G. Dimitrakopoulos, P. Demestichas, Intelligent transportation systems, IEEE Veh. Technol. Mag. 5 (1) (2010) 77-84.

[15] J. Zhang, F.-Y. Wang, K. Wang, W.-H. Lin, X. Xu, C. Chen, Data-driven intelligent transportation systems: a survey, IEEE Trans. Intell. Transp. Syst. 12 (4) (2011) 1624-1639.

[16] S. Al-Sultan, M.M. Al-Doori, A.H. Al-Bayatti, H. Zedan, A comprehensive survey on vehicular ad hoc network, J. Netw. Comput. Appl. 37 (2014) 380-392.

[17] D. Jiang, L. Delgrossi, IEEE 802.11p: towards an international standard for wireless access in vehicular environments, in: IEEE Vehicular Technology Conference, VTC Spring, IEEE Computer Society, 2008, pp. 2036-2040.

[18] R.A. Uzcategui, A.J.D. Sucre, G. Acosta-Marum, WAVE: a tutorial, IEEE Commun. Mag. 47 (5) (2009) 126-133.

[19] B.T. Sharef, R.A. Alsaqour, M. Ismail, Vehicular communication ad hoc routing protocols: a survey, J. Netw. Comput. Appl. 40 (2014) 363-396.

[20] D. Milojicic, F. Douglis, R. Wheeler, Mobility: Processes, Computers, and Agents, ACM, 1999.

[21] R. Trillo, S. Ilarri, E. Mena, Comparison and performance evaluation of mobile agent platforms, in: Third International Conference on Autonomic and Autonomous Systems, ICAS, IEEE Computer Society, 2007, pp. 41-46.

[22] O. Urra, S. Ilarri, E. Mena, Agents jumping in the air: dream or reality?, in: 10th International Work-Conference on Artificial Neural Networks (IWANN), Special Session on Practical Applications of Agents and Multi-Agent Systems, Springer, 2009, pp. 627-634 
[23] S. Ilarri, R. Trillo-Lado, E. Mena, SPRINGS: a scalable platform for highly mobile agents in distributed computing environments, in: Fourth International WoWMoM 2006 Workshop on Mobile Distributed Computing, MDC, IEEE Computer Society, 2006, pp. 633-637.

[24] S. Ilarri, P. Roig, R. Trillo-Lado, GeoSPRINGS: towards a location-aware mobile agent platform, in: 16th International Symposium on Web and Wireless Geographical Information Systems, W2GIS, in: Lecture Notes in Computer Science (LNCS), vol. 10819, Springer, 2018, pp. 51-60.

[25] O. Urra, S. Ilarri, Using mobile agents in vehicular networks for data processing, in: 14th International Conference on Mobile Data Management (MDM), Ph.D. Forum, vol. 2, IEEE Computer Society, 2013, pp. 11-14.

[26] A. Boubrima, W. Bechkit, H. Rivano, Optimal WSN deployment models for air pollution monitoring, IEEE Trans. Wirel. Commun. 16 (5) (2017) 2723-2735.

[27] M. Bacco, F. Delmastro, E. Ferro, A. Gotta, Environmental monitoring for smart cities, IEEE Sens. J. 17 (23) (2017) 7767-7774.

[28] C.T. Calafate, K. Cicenia, O. Alvear, J.C. Cano, P. Manzoni, Estimating rainfall intensity by using vehicles as sensors, in: Wireless Days, IEEE Computer Society, 2017, pp. 21-26.

[29] C. Lochert, B. Scheuermann, M. Caliskan, M. Mauve, The feasibility of information dissemination in vehicular ad-hoc networks, in: Fourth Annual Conference on Wireless on Demand Network Systems and Services, WONS, 2007, pp. 92-99.

[30] T. Delot, N. Mitton, S. Ilarri, T. Hien, GeoVanet: a routing protocol for query processing in vehicular networks, Mob. Inf. Syst. 7 (4) (2011) 329-359.

[31] O. Urra, S. Ilarri, MAVSIM: testing VANET applications based on mobile agents, in: CRC Taylor and Francis Group, 2016, pp. 199-224, Ch. 10

[32] Y. Zhao, Q. Han, Spatial crowdsourcing: current state and future directions, IEEE Commun. Mag. 54 (7) (2016) 102-107.

[33] OpenStreetMap Foundation (OSMF), Openstreetmap, http://openstreetmap.org, 2019, last access on February 6.

[34] M. Ruiz, L. Velasco, Background, 1st edition, Wiley Publishing, 2017, pp. 15-42, Ch. 2 .

[35] T. Darwish, K.A. Bakar, Traffic density estimation in vehicular ad hoc networks: a review, Ad Hoc Netw. 24 (2015) 337-351.

[36] B.S. Kerner, Introduction to Modern Traffic Flow Theory and Control, Springer, 2009.

[37] F.J. Martinez, M. Fogue, C.K. Toh, J.-C. Cano, C.T. Calafate, P. Manzoni, Computer simulations of VANETs using realistic city topologies, Wirel. Pers. Commun. 69 (2) (2012) 639-663.

[38] J. Barrachina, P. Garrido, M. Fogue, F.J. Martinez, J.-C. Cano, C.T. Calafate, P. Manzoni, Road side unit deployment: a density-based approach, IEEE Trans. Intell. Transp. Syst. 5 (3) (2013) 30-39.

[39] R.K. Ganti, F. Ye, H. Lei, Mobile crowdsensing: current state and future challenges, IEEE Commun. Mag. 49 (11) (2011) 32-39.

[40] X. Sheng, J. Tang, W. Zhang, Energy-efficient collaborative sensing with mobile phones, in: 31st Annual IEEE International Conference on Computer Communications, INFOCOM, 2012, pp. 1916-1924.

[41] G. Cardone, A. Cirri, A. Corradi, L. Foschini, R. Ianniello, R. Montanari, Crowdsensing in urban areas for city-scale mass gathering management: geofencing and activity recognition, IEEE Sens. J. 14 (12) (2014) 4185-4195.

[42] Z. Chen, R. Fu, Z. Zhao, Z. Liu, L. Xia, L. Chen, P. Cheng, C.C. Cao, Y. Tong, C.J. Zhang, gMission: a general spatial crowdsourcing platform, Proc. VLDB Endow. 7 (13) (2014) 1629-1632.

[43] H. To, G. Ghinita, C. Shahabi, A framework for protecting worker location privacy in spatial crowdsourcing, Proc. VLDB Endow. 7 (10) (2014) 919-930.

[44] B. Guo, Z. Wang, Z. Yu, Y. Wang, N.Y. Yen, R. Huang, X. Zhou, Mobile crowd sensing and computing: the review of an emerging human-powered sensing paradigm, ACM Comput. Surv. 48 (1) (2015) 7.

[45] Y. Tong, L. Chen, C. Shahabi, Spatial crowdsourcing: challenges, techniques, and applications, Proc. VLDB Endow. 10 (12) (2017) 1988-1991.

[46] Y. Tong, J. She, B. Ding, L. Wang, L. Chen, Online mobile micro-task allocation in spatial crowdsourcing, in: 32nd International Conference on Data Engineering, ICDE, IEEE Computer Society, 2016, pp. 49-60.

[47] H. To, C. Shahabi, L. Kazemi, A server-assigned spatial crowdsourcing framework, ACM Trans. Spat. Algorithms Syst. 1 (1) (2015) 2.

[48] P. Cheng, X. Lian, Z. Chen, R. Fu, L. Chen, J. Han, J. Zhao, Reliable diversity-based spatial crowdsourcing by moving workers, Proc. VLDB Endow. 8 (10) (2015) $1022-1033$

[49] U. ul Hassan, E. Curry, Efficient task assignment for spatial crowdsourcing: a combinatorial fractional optimization approach with semi-bandit learning, Expert Syst. Appl. 58 (2016) 36-56.

[50] H. To, Task assignment in spatial crowdsourcing: challenges and approaches, in: Third ACM SIGSPATIAL PhD Symposium, ACM, 2016, 1.

[51] Y. Wang, H. Li, T. Li, Participant selection for data collection through deviceto-device communications in mobile sensing, Pers. Ubiquitous Comput. 21 (1) (2017) 31-41.

[52] E. Wang, Y. Yang, J. Wu, W. Liu, X. Wang, An efficient prediction-based user recruitment for mobile crowdsensing, IEEE Trans. Mob. Comput. 17 (1) (2018) 16-28.
[53] S. Abdelhamid, H.S. Hassanein, G. Takahara, Reputation-aware, trajectory-based recruitment of smart vehicles for public sensing, IEEE Trans. Intell. Transp. Syst. 19 (5) (2018) 1387-1400.

[54] R. Azzam, R. Mizouni, H. Otrok, A. Ouali, S. Singh, GRS: a group-based recruitment system for mobile crowd sensing, J. Netw. Comput. Appl. 72 (2016) 38-50.

[55] N. Ta, G. Li, T. Zhao, J. Feng, H. Ma, Z. Gong, An efficient ride-sharing framework for maximizing shared route, IEEE Trans. Knowl. Data Eng. 30 (2) (2018) 219-233.

[56] A.S. Fonteles, S. Bouveret, J. Gensel, A programming framework for spatial crowdsourcing, in: 15th International Conference on Advances in Mobile Computing \& Multimedia, MoMM, ACM, 2017, pp. 131-140.

[57] H. Qiu, J. Chen, S. Jain, Y. Jiang, M. McCartney, G. Kar, F. Bai, D.K. Grimm, M. Gruteser, R. Govindan, Towards robust vehicular context sensing, IEEE Trans. Veh. Technol. 67 (3) (2018) 1909-1922.

[58] J. Yu, K.H. Low, A. Oran, P. Jaillet, Hierarchical Bayesian nonparametric approach to modeling and learning the wisdom of crowds of urban traffic route planning agents, in: 2012 IEEE/WIC/ACM International Conferences on Web Intelligence and Intelligent Agent Technology (WI-IAT), vol. 2, 2012, pp. 478-485.

[59] F. Shi, Z. Qin, J.A. McCann, OPPay: design and implementation of a payment system for opportunistic data services, in: 37th International Conference on Distributed Computing Systems, ICDCS, IEEE Computer Society, 2017, pp. 1618-1628.

[60] T. Dimitriou, I. Krontiris, Privacy-respecting auctions and rewarding mechanisms in mobile crowd-sensing applications, J. Netw. Comput. Appl. 100 (2017) 24-34.

[61] O. Wolfson, B. Xu, P. Sistla, An economic model for resource exchange in mobile peer to peer networks, in: 16th International Conference on Scientific and Statistical Database Management, SSDBM, IEEE Computer Society, 2004, pp. 235-244

[62] L.-Y. Yeh, Y.-C. Lin, A proxy-based authentication and billing scheme with incentive-aware multihop forwarding for vehicular networks, IEEE Trans. Intell. Transp. Syst. 15 (4) (2014) 1607-1621.

[63] L.G. Jaimes, I.J. Vergara-Laurens, A. Raij, A survey of incentive techniques for mobile crowd sensing, IEEE Int. Things J. 2 (5) (2015) 370-380.

[64] H. Gao, C.H. Liu, W. Wang, J. Zhao, Z. Song, X. Su, J. Crowcroft, K.K. Leung, A survey of incentive mechanisms for participatory sensing, IEEE Commun. Surv. Tutor. 17 (2) (2015) 918-943.

[65] X. Zhang, Z. Yang, W. Sun, Y. Liu, S. Tang, K. Xing, X. Mao, Incentives for mobile crowd sensing: a survey, IEEE Commun. Surv. Tutor. 18 (1) (2016) 54-67.

[66] B. Di, T. Wang, L. Song, Z. Han, Collaborative smartphone sensing using overlapping coalition formation games, IEEE Trans. Mob. Comput. 16 (1) (2017) 30-43.

[67] U. Lee, M. Gerla, A survey of urban vehicular sensing platforms, Comput. Netw. 54 (4) (2010) 527-544.

[68] J.R. Vacca, Handbook of Sensor Networking: Advanced Technologies and Applications, CRC Press, 2015.

[69] L. Liu, W. Wei, D. Zhao, H. Ma, Urban resolution: new metric for measuring the quality of urban sensing, IEEE Trans. Mob. Comput. 14 (12) (2015) 2560-2575.

[70] Y. Wang, J. Jiang, T. Mu, Context-aware and energy-driven route optimization for fully electric vehicles via crowdsourcing, IEEE Trans. Intell. Transp. Syst. 14 (3) (2013) 1331-1345.

[71] P.M. Santos, T. Calcada, D. Guimarães, T. Condeixa, S. Sargento, A. Aguiar, J.a. Barros, Demo: platform for collecting data from urban sensors using vehicular networking, in: 21st Annual International Conference on Mobile Computing and Networking, MobiCom, ACM, 2015, pp. 167-169.

[72] D. Cerotti, S. Distefano, G. Merlino, A. Puliafito, A crowd-cooperative approach for intelligent transportation systems, IEEE Trans. Intell. Transp. Syst. 18 (6) (2016) 1529-1539.

[73] A. Grazioli, M. Picone, F. Zanichelli, M. Amoretti, Collaborative mobile application and advanced services for smart parking, in: 14th International Conference on Mobile Data Management (MDM), vol. 2, IEEE Computer Society, 2013 pp. 39-44.

[74] D. Asprone, S. Di Martino, P. Festa, Improving sensing coverage of probe vehicles with probabilistic routing, in: M.R. Luaces, F. Karimipour (Eds.), 16th International Symposium on Web and Wireless Geographical Information Systems, W2GIS, Springer, 2018, pp. 1-10.

[75] D. Zhao, H. Ma, Q. Li, S. Tang, A unified delay analysis framework for opportunistic data collection, Wirel. Netw. 24 (4) (2018) 1313-1325.

[76] X. Liu, X. Chen, X. Xu, E. Mai, H.Y. Noh, P. Zhang, L. Zhang, Delay effect in mobile sensing system for urban air pollution monitoring, in: 15th ACM Conference on Embedded Network Sensor Systems, SenSys, ACM, 2017, 73.

[77] X. Wang, X. Zheng, Q. Zhang, T. Wang, D. Shen, Crowdsourcing in ITS: the state of the work and the networking, IEEE Trans. Intell. Transp. Syst. 17 (6) (2016) 1596-1605.

[78] C. Zuo, K. Liang, Z.L. Jiang, J. Shao, J. Fang, Cost-effective privacy-preserving vehicular urban sensing system, Pers. Ubiquitous Comput. 21 (5) (2017) 893-901.
67

68

69

70

71

72

73

74

75 